\title{
Implicit preconditioned WENO scheme for steady viscous flow computation
}

\author{
Juan-Chen Huang ${ }^{\mathrm{a}}$, Herng Lin ${ }^{\mathrm{b}}$, Jaw-Yen Yang ${ }^{\mathrm{c}, *}$ \\ a Department of Merchant Marine, National Taiwan Ocean University, 2 Pei-Ning Road, Keelung 20224, Taiwan \\ ${ }^{\mathrm{b}}$ Chung Shan Institute of Science and Technology, Lung-Tan Box 90008-15-5, Tauyung 32526, Taiwan \\ ${ }^{\mathrm{c}}$ Institute of Applied Mechanics, National Taiwan University, 1, Sec. 4, Roosevelt Road, Taipei 10764, Taiwan
}

\section{A R T I C L E I N F O}

\section{Article history:}

Received 31 March 2008

Received in revised form 2 September 2008

Accepted 21 September 2008

Available online 2 October 2008

\section{Keywords:}

Preconditioned Navier-Stokes equations WENO schemes

Low Mach number flow

Transonic flows

Hypersonic flow

All speeds

Spalart-Allmaras turbulence model

\begin{abstract}
A B S T R A C T
A class of lower-upper symmetric Gauss-Seidel implicit weighted essentially nonoscillatory (WENO) schemes is developed for solving the preconditioned Navier-Stokes equations of primitive variables with Spalart-Allmaras one-equation turbulence model. The numerical flux of the present preconditioned WENO schemes consists of a first-order part and high-order part. For first-order part, we adopt the preconditioned Roe scheme and for the high-order part, we employ preconditioned WENO methods. For comparison purpose, a preconditioned TVD scheme is also given and tested. A time-derivative preconditioning algorithm is devised and a discriminant is devised for adjusting the preconditioning parameters at low Mach numbers and turning off the preconditioning at intermediate or high Mach numbers. The computations are performed for the two-dimensional lid driven cavity flow, low subsonic viscous flow over S809 airfoil, three-dimensional low speed viscous flow over 6:1 prolate spheroid, transonic flow over ONERA-M6 wing and hypersonic flow over HB-2 model. The solutions of the present algorithms are in good agreement with the experimental data. The application of the preconditioned WENO schemes to viscous flows at all speeds not only enhances the accuracy and robustness of resolving shock and discontinuities for supersonic flows, but also improves the accuracy of low Mach number flow with complicated smooth solution structures.
\end{abstract}

(c) 2008 Elsevier Inc. All rights reserved.

\section{Introduction}

It is well known that most of the numerical algorithms developed for compressible flows are often inefficient or even inaccurate at low Mach numbers. When the flow Mach number becomes relatively small in comparison with the acoustic speed, the convective terms of the time dependent system of equations become stiff and this may deteriorate the convergence when time marching schemes are used. Also, the pressure gradient term in momentum equation becomes singular and may increase roundoff error [1]. For the type of low Mach number flow, that the velocity in entire flowfield is low, the stiffness problems can be avoided by solving incompressible equations instead. But for the type of low Mach number flow contains some regions of incompressible flow while other regions of compressible flow, extending the solver for compressible equations to low Mach number flows seems to be a viable choice. The basic principle of extending compressible algorithms to incompressible flow limit is to precondition the usual compressible Navier-Stokes equations to overcome the mentioned difficulties. Convergence can be made independent of Mach number by altering the acoustic speeds of the system such that all eigenvalues become of the same order. Multiplying the preconditioning matrix to normalize the system

\footnotetext{
* Corresponding author. Tel.: +886 23366 5636; fax: +886 223629290.

E-mail address: yangiy@iam.ntu.edu.tw (J.-Y. Yang).
} 
eigenvalues is a successful and popular approach. Over the past two decades, a number of preconditioning methods have been proposed [2-7].

The weighted essentially nonoscillatory (WENO) schemes proposed by Liu et al. [8] and extended by Jiang and Shu [9] can achieve good convergence property while keeping robustness and high-order accuracy of ENO schemes. The WENO schemes have been quite successful in applications, especially for problems containing both shocks and complicated smooth solution structures. For low speed incompressible flow, a class of implicit WENO schemes has been successfully applied to incompressible flow problems by Yang et al. [10] based on artificial compressibility formulation. Good convergence rate to a steady-state solution has been illustrated. Yang et al. [11] also proposed a method which adopts a numerical flux in flux limiter form for the WENO spatial operator for convective flux that allows for more flexibility to implement various first-order entropy satisfying dissipative schemes.

In this paper, we present a high-order accurate WENO scheme for solving the preconditioned Navier-Stokes equations. This scheme is a direct extension of a WENO scheme of flux limiter form by Yang et al. [11,12], which has been successfully applied to 2D/3D aerodynamic problems. Following the procedures proposed by Weiss and Smith [4], the preconditioning matrix is derived. We also introduced a discriminant for adjusting the preconditioning parameters at low Mach numbers and turning off the preconditioning at intermediate or high Mach numbers. For turbulent flow calculations, Spalart-Allmaras one-equation turbulence model [13] simplified by dropping the transition terms is adopted. The model provides a desirable tool for numerical computation of flow involving complex geometry. The performance of this model has been tested. To improve the efficiency and convergence to steady state, the lower-upper symmetric Gauss-Seidel (LU-SGS) implicit algorithm is adopted. We apply the resulting implicit preconditioned WENO schemes to compute several aerodynamic flows with wide speed range to test both the convergence rate and the accuracy of the methods. The main contribution of the present work is to validate the WENO scheme applied to the preconditioned Navier-Stokes equations, and to point out the efficiency of computation over a wide range of Mach numbers. The preconditioned Navier-Stokes equations are stated in Section 2 and the basis of the numerical scheme are presented in Section 3. In Section 4, the computational results with a wide range of freestream Mach numbers in both two and three space dimensions are presented to validate the effectiveness of the present method. Some concluding remarks are given in Section 5.

\section{Governing equation}

\subsection{The Navier-Stokes equations}

The non-dimensional unsteady Reynolds averaged Navier-Stokes equations with the Spalart-Allmaras one-equation turbulence model in general curvilinear coordinates $(\xi, \eta, \zeta)$ system are expressed as

$$
\frac{\partial \hat{Q}}{\partial t}+\frac{\partial\left(\hat{E}-\hat{E}_{v}\right)}{\partial \xi}+\frac{\partial\left(\hat{F}-\hat{F}_{v}\right)}{\partial \eta}+\frac{\partial\left(\hat{G}-\hat{G}_{v}\right)}{\partial \zeta}=\hat{H},
$$

where $t$ is time, $\hat{Q}$ is the vector of conservative variables, $\hat{E}, \hat{F}$ and $\hat{G}$ are convective flux vectors, $\hat{E}_{v}, \hat{F}_{v}$ and $\hat{G}_{v}$ are diffusive flux vectors in $\xi, \eta, \zeta$ direction, respectively. The source vector defined as $\hat{H}=\left[0,0,0,0,0, H_{6}\right]^{T}$, where $H_{6}$ is the source term associated with turbulence eddy viscosity production, conservative and nonconservative diffusion and near wall turbulence destruction [14]. Here, only the fluxes in the $\xi$ direction are shown as

$$
\begin{aligned}
& \hat{Q}=\frac{1}{J}[\rho, \rho u, \rho v, \rho w, e, \widetilde{v}]^{T}, \\
& \hat{E}=\frac{1}{J}\left[\rho U, \rho u U+\xi_{x} p, \rho v U+\xi_{y} p, \rho w U+\xi_{z} p,(e+p) U, \widetilde{v} U\right]^{T}, \\
& \hat{E}_{v}=\frac{1}{\operatorname{Re}_{\infty} J}\left[0, E_{v 2}, E_{v 3}, E_{v 4}, E_{v 5}, E_{v 6}\right]^{T},
\end{aligned}
$$

where the elements $E_{v 2}, E_{v 3}, E_{v 4}, E_{v 5}$ and $E_{v 6}$ are defined as

$$
\begin{aligned}
& E_{v 2}=\tau_{x x} \xi_{x}+\tau_{x y} \xi_{y}+\tau_{x z} \xi_{z}, \\
& E_{v 3}=\tau_{x y} \xi_{x}+\tau_{y y} \xi_{y}+\tau_{y z} \xi_{z}, \\
& E_{v 4}=\tau_{x z} \xi_{x}+\tau_{y z} \xi_{y}+\tau_{z z} \xi_{z}, \\
& E_{v 5}=u E_{v 2}+v E_{v 3}+w E_{v 4}+q_{x} \xi_{x}+q_{y} \xi_{y}+q_{z} \xi_{z}, \\
& E_{v 6}=\frac{1}{\sigma_{\varepsilon}}\left[\frac{\mu_{\ell}}{\rho}+\left(1+C_{B 2}\right) \widetilde{v}\right]\left(\frac{\partial \widetilde{v}}{\partial x} \xi_{x}+\frac{\partial \widetilde{v}}{\partial y} \xi_{y}+\frac{\partial \widetilde{v}}{\partial z} \xi_{z}\right) .
\end{aligned}
$$

Similar expressions can be given for the fluxes in the $\eta$ and $\zeta$ directions. In the above equations, $J$ is the Jacobian of coordinate transform, $\rho$ is density, $u, v$ and $w$ are velocity components in $x, \mathrm{y}$ and $z$ direction, respectively, $e$ is total energy per unit volume. The static pressure $p$ is related to the dependent variables by the equation of state for a perfect gas $p=(\gamma-1)\left[e-\rho\left(u^{2}+v^{2}+w^{2}\right) / 2\right]$, where $\gamma$ is the ratio of specific heats. $q_{x}, q_{y}, q_{z}$ are heat fluxes in $x, y$ and $z$ direction, respectively. $U=u \xi_{x}+v \xi_{y}+w \xi_{z}$ is contravariant velocity in $\xi$ direction, $\tau_{x x}, \tau_{x y}, \tau_{x z}, \tau_{y y}, \tau_{y z}, \tau_{z z}$ are shear stresses. $\mu_{\ell}$ is molecular 
viscosity, which is calculated by Sutherland's law in present study. $\widetilde{v}$ is the turbulent variable in the Spalart-Allmaras oneequation model, $\sigma_{\varepsilon}$ and $C_{B 2}$ are empirical constants. The detailed form of the source terms and empirical constants for turbulent equation can be found in Refs. $[13,14]$. The equations are non-dimensionalized by characteristic length $\bar{L}$, freestream velocity $\bar{V}_{\infty}$ and other reference quantities as follows:

$$
\begin{aligned}
& x=\bar{x} / \bar{L}, \quad t=\bar{t} \bar{V}_{\infty} / \bar{L}, \quad \rho=\bar{\rho} / \bar{\rho}_{\infty}, \quad u=\bar{u} / \bar{V}_{\infty}, \\
& p=\bar{p} / \bar{\rho}_{\infty} \bar{V}_{\infty}^{2}, \quad T=\bar{T} / \bar{T}_{\infty}, \quad \mu_{\ell}=\bar{\mu}_{\ell} / \bar{\mu}_{\ell \infty}, \quad \widetilde{v}=\bar{\rho}_{\infty} \overline{\widetilde{v}} / \bar{\mu}_{\ell \infty} .
\end{aligned}
$$

\subsection{The preconditioned system}

The preconditioned version of Eq. (1) is given as

$$
\Gamma \frac{\partial \hat{Q}_{p}}{\partial t}+\frac{\partial \hat{E}}{\partial \xi}+\frac{\partial \hat{F}}{\partial \eta}+\frac{\partial \hat{G}}{\partial \zeta}=\frac{\partial \hat{E}_{v}}{\partial \xi}+\frac{\partial \hat{F}_{v}}{\partial \eta}+\frac{\partial \hat{G}_{v}}{\partial \zeta}+\hat{H}
$$

where $\hat{Q}_{p}=[p, u, v, w, T, \widetilde{v}] / J$ is the vector of primitive variables. The preconditioning matrix $\Gamma$ defined by Weiss and Smith [4] was given as

$$
\Gamma=\left(\begin{array}{cccccc}
\Theta & 0 & 0 & 0 & \rho_{T} & 0 \\
\Theta u & \rho & 0 & 0 & \rho_{T} u & 0 \\
\Theta v & 0 & \rho & 0 & \rho_{T} u & 0 \\
\Theta w & 0 & 0 & \rho & \rho_{T} w & 0 \\
\Theta H_{0}-1 & \rho u & \rho v & \rho w & \rho_{T} H_{0}+\rho C_{p} & 0 \\
0 & 0 & 0 & 0 & 0 & 1
\end{array}\right)
$$

with

$$
\Theta=\frac{1}{V_{r}^{2}}-\frac{\rho_{T}}{\rho C_{p}} .
$$

It is noted that the transport equation of turbulent variable is not preconditioned in our work. Hence, elements of the 6th column and 6th row in preconditioning matrix are zeros except for the diagonal ones. In Eqs. (3) and (4), $H_{0}$ is the total enthalpy, $C_{p}=1 /(\gamma-1) M_{\infty}^{2}$ is constant-pressure specific heat and $\rho_{T}=\partial \rho / \partial T, V_{r}=M_{r} c$ is reference velocity, $c$ is local sonic speed and $M_{r}$ is a reference Mach number, defined as

$$
M_{r}=\min \left[1, \max \left(M, M_{r, \min }\right)\right],
$$

where $M$ is Mach number of local flowfield, $M_{r, \min }$ is the minimum value of reference Mach number $M_{r}$, which is defined by Weiss and Smith [4] to prevent $\Theta$ from diverging and its value is $M_{r \text {,min }}=10^{-5}$. Refs. [5,6] proposed the following formula

$$
M_{r, \min }^{2}=3 M_{\infty}^{2}
$$

which is a function of freestream Mach number and is used in OVERFLOW code. In Ref. [7], Edwards and Roy proposed the following formula for the reference velocity

$$
V_{r}^{2}=\min \left[c^{2}, \max \left(|V|^{2}, K\left|V_{\infty}\right|^{2}\right)\right],
$$

where $V$ is local velocity and $V_{\infty}$ is freestream velocity, and the parameter $K=0.5$ was suggested. Turkel [2] suggested that $K=0.4$ for inviscid flow and $K=1.0$ for viscous flow. He also pointed out that $K$ can be setting up to 3.0 in some cases if necessary. In the present study, we summarize the above experiences and propose the rules for choosing $M_{r, m i n}$ values as follows:

$$
M_{r, \text { min }}^{2}=\left\{\begin{array}{lll}
3 M_{\infty}^{2} & \text { if } & M_{\infty} \leqslant 0.05 \\
0.15 M_{\infty} & \text { if } \quad 0.05 \leqslant M_{\infty} \leqslant 0.3, \\
0.5 M_{\infty}^{2} & \text { if } \quad M_{\infty} \geqslant 0.3 .
\end{array}\right.
$$

For viscous flow computations, Weiss and Smith [4] pointed out that the reference velocity $V_{r}$ must be larger than the parameter of viscous velocity $v /\left(\operatorname{Re}_{\infty} \Delta x\right)$, where $v$ is local kinematic viscosity (including turbulent effects), $\Delta x$ is the length scale of grid, representing the diffusion phenomenon. Here, we set $\Delta x$ equal to the shortest cell length among three directions of a grid cell. In viscous flow computation, the reference Mach number is written as

$$
M_{r}=\max \left\{\min \left[1, \max \left(M, M_{r, \min }\right)\right], M_{r, v i s}\right\},
$$

where $M_{r, v i s}=v /\left(\operatorname{Re}_{\infty} c \Delta x\right)$. The flux Jacobian matrix of the preconditioned system is $A_{\Gamma}=\Gamma^{-1} A_{p}$, where $A_{p}$ is defined as $\partial \hat{E} / \partial \hat{Q}_{p}$. The inverse of the preconditioning matrix $\Gamma^{-1}$ could be obtained by some symbolic operations. The flux Jacobian matrix $\hat{A}_{\Gamma}$ is given as 


$$
A_{\Gamma}=\left[\begin{array}{cccccc}
\left(\rho C_{p}+\rho_{T}\left(H_{0}-q^{2}\right)\right) / d^{\prime} & \rho_{T} u / d^{\prime} & \rho_{T} v / d^{\prime} & \rho_{T} w / d^{\prime} & -\rho_{T} / d^{\prime} & 0 \\
\kappa_{x} / \rho & \bar{u} & 0 & 0 & 0 & 0 \\
\kappa_{y} / \rho & 0 & \bar{u} & 0 & 0 & 0 \\
\kappa_{z} / \rho & 0 & 0 & \bar{u} & 0 & 0 \\
\left(1+0.5 \Theta q^{2}\right) / d^{\prime} & \Theta u / d^{\prime} & \Theta v / d^{\prime} & \Theta w / d^{\prime} & -\Theta / d^{\prime} & 0 \\
0 & 0 & 0 & 0 & 0 & \bar{u}
\end{array}\right],
$$

where

$$
d^{\prime}=\rho C_{p} \Theta+\rho_{T}=\frac{\rho}{\left[(\gamma-1) T M_{r}^{2}\right]} .
$$

The eigenvalues of matrix $A_{\Gamma}$ are

$$
\begin{aligned}
& \lambda_{1}=\lambda_{2}=\lambda_{3}=\lambda_{6}=\bar{u}, \quad \lambda_{4}=u^{\prime}+c^{\prime}, \quad \lambda_{5}=u^{\prime}-c^{\prime} . \\
& u \prime=\bar{u}\left(1+M_{r}^{2}\right) / 2 \quad \text { and } \quad c^{\prime}=\sqrt{\bar{u}^{2}\left(1-M_{r}^{2}\right)+4 M_{r}^{2} c^{2}} / 2 .
\end{aligned}
$$

where $\bar{u}$ is defined as $u k_{x}+v k_{y}+w k_{z}$, and $\left(k_{x}, k_{y}, k_{z}\right)=\nabla k /|\nabla k|, k=\xi, \eta, \zeta$ is a unit vector. The eigenvalues will be degenerated automatically to the non-preconditioned system at $M_{r}=1$. Since the non-dimensional quantities are different between current paper and Pandya et al. [6], the matrices of right and left eigenvectors of $A_{\Gamma}$ are shown here,

$$
\begin{aligned}
R & =\left(\begin{array}{cccccc}
0 & 0 & 0 & c_{5} / 2 c^{\prime} & c_{4} / 2 c^{\prime} & 0 \\
0 & -k_{z} & k_{y} & k_{x} / 2 \rho c^{\prime} & -k_{x} / 2 \rho c^{\prime} & 0 \\
k_{z} & 0 & -k_{x} & k_{y} / 2 \rho c^{\prime} & -k_{y} / 2 \rho c^{\prime} & 0 \\
-k_{y} & k_{x} & 0 & k_{z} / 2 \rho c^{\prime} & -k_{z} / 2 \rho c^{\prime} & 0 \\
k_{x} & k_{y} & k_{z} & c_{5} / 2 \rho C_{p} c^{\prime} & c_{4} / 2 \rho C_{p} c^{\prime} & 0 \\
0 & 0 & 0 & 0 & 0 & 1
\end{array}\right), \\
L & =\left(\begin{array}{cccccc}
-k_{x} / \rho C_{p} & 0 & k_{z} & -k_{y} & k_{x} & 0 \\
-k_{y} / \rho C_{p} & -k_{z} & 0 & k_{x} & k_{y} & 0 \\
-k_{z} / \rho C_{p} & k_{y} & -k_{x} & 0 & k_{z} & 0 \\
1 & \rho c_{4} k_{x} & \rho c_{4} k_{y} & \rho c_{4} k_{z} & 0 & 0 \\
1 & -\rho c_{5} k_{x} & -\rho c_{5} k_{y} & -\rho c_{5} k_{z} & 0 & 0 \\
0 & 0 & 0 & 0 & 0 & 1
\end{array}\right) .
\end{aligned}
$$

The parameters $c_{4}$ and $c_{5}$ in above equations are defined as

$$
c_{4}=u^{\prime}+c^{\prime}+M_{r}^{2} \bar{u} \quad \text { and } \quad c_{5}=-u^{\prime}+c^{\prime}+M_{r}^{2} \bar{u} .
$$

\section{Numerical method}

\subsection{Spatial discretization}

A semi-discrete finite volume method is used to ensure that the final converged solution is independent of the integration procedure and to avoid metric singularity problems. The finite volume method is based on the local flux balance of each mesh cell. The semi-discrete form of Eq. (2) can be written as

$$
\Gamma_{i, j, k} \frac{\left(\hat{Q}_{p}^{n+1}-\hat{Q}_{p}^{n}\right)_{i, j, k}}{\Delta t}=-\left[\widetilde{E}_{i+\frac{1}{2}, j, k}-\widetilde{E}_{i-\frac{1}{2}, j, k}\right]^{n+1}-\left[\widetilde{F}_{i, j+\frac{1}{2}, k}-\widetilde{F}_{i, j-\frac{1}{2}, k}\right]^{n+1}-\left[\widetilde{G}_{i, j, k+\frac{1}{2}}-\widetilde{G}_{i, j, k-\frac{1}{2}}\right]^{n+1}+\hat{H}_{i, j, k}^{n+1},
$$

where $(i, j, k)$ is the control point of finite volume. The spatial differencing adopts conservative upwind schemes for the inviscid convective fluxes $(\widetilde{E}, \widetilde{F}, \widetilde{G})$ and second-order central differencing for viscous flux $\left(\widetilde{E}_{v}, \widetilde{F}_{v}, \widetilde{G}_{v}\right)$. Weiss and Smith $[4]$ proposed the Roe's scheme in preconditioned system. The numerical flux at cell interface $i+1 / 2$ in direction $i$ is written as

$$
\widetilde{E}_{i+1 / 2}^{\text {P-Roe }}=\frac{1}{2}\left[\hat{E}_{i}+\hat{E}_{i+1}+\Gamma_{i+1 / 2} R_{i+1 / 2}\left|\hat{\Lambda}_{i+1 / 2}\right| L_{i+1 / 2}\left(Q_{p, i+1}-Q_{p, i}\right)\right] .
$$

The matrix of right and left eigenvectors $R$ and $L$, the diagonal matrix of eigenvalues $\hat{\Lambda}$, and $\Gamma$ are evaluated by the primitive flow parameters, including density $\rho$, velocities $u, v, w$ and total enthalpy $H_{0}$, which are defined at cell interface and are evaluated by the formulas of Roe's averages [15].

To manifest the characteristics of WENO schemes in preconditioned system, we also extend the symmetric TVD scheme by Yee [16] for the Euler equations to the present preconditioned Navier-Stokes equations for comparison purpose. The sym- 
metric TVD, WENO2 and WENO3 schemes in preconditioned system which designated as P-STVD, P-WENO2 and P-WENO3 are derived as follows.

\subsubsection{P-STVD scheme}

Referring to the original TVD scheme of Yee [16], the numerical flux at cell interface $i+1 / 2$ for the present preconditioned second-order symmetric TVD scheme can be written as

$$
\widetilde{E}_{i+1 / 2}^{P-S T V D}=\frac{1}{2}\left[\hat{E}_{i}+\hat{E}_{i+1}+\Gamma_{i+1 / 2} R_{i+1 / 2} \Phi_{i+1 / 2}\left(\frac{|\nabla \xi|}{J}\right)_{i+1 / 2}\right],
$$

where $\Phi_{i+1 / 2}$ is modified flux vector [16]. In this work, the entropy parameter is defined as $\delta=\beta_{e} \times \max \left(|\bar{u}|,\left|\bar{u}^{\prime}+c^{\prime}\right|,\left|\bar{u}^{\prime}-c^{\prime}\right|\right)$, which equals a factor $\beta_{e}$ times the maximum absolute eigenvalue. The factor $\beta_{e}$ is selected to be under 0.005 for low Mach number flow $\left(M_{\infty}<0.1\right), 0.01$ to 0.1 for subsonic or transonic flow $\left(0.1<M_{\infty}<1.0\right)$ and $0.1-0.5$ for supersonic or hypersonic flow $\left(M_{\infty}>1.0\right)$.

\subsubsection{P-WENO2 scheme}

Next, we reformulate the new type WENO scheme used for compressible flow computations proposed by Yang et al. [11] to implement the preconditioned system. A P-WENO2 numerical flux at a cell surface $i+1 / 2$ in direction $i$ can be defined by

$$
\tilde{E}_{i+1 / 2}^{\text {P-WENO2 }}=\Gamma_{i+1 / 2}\left(\tilde{E}_{i+1 / 2}^{L}+\tilde{E}_{i+1 / 2}^{H 2}\right)=\tilde{E}_{i+1 / 2}^{\text {P-Roe }}+\Gamma_{i+1 / 2} \tilde{E}_{i+1 / 2}^{H 2},
$$

where $\widetilde{E}_{i+1 / 2}^{L}$ is the numerical flux of a first-order dissipative entropy satisfying scheme. Here the preconditioned Roe scheme is adopted. $\widetilde{E}_{i+1 / 2}^{H 2}$ is a high-order flux with P-WENO2 limiter, defined as

$$
\tilde{E}_{i+1 / 2}^{H 2}=\sum_{s=1}^{5} \tilde{E}_{i+1 / 2, s}^{H 2} r_{s} .
$$

The sth element of $\widetilde{E}_{i+1 / 2}^{H 2}$ is

$$
\tilde{E}_{i+1 / 2, s}^{H 2}=\frac{1}{2}\left[\omega_{0, s}^{+} \Delta E_{i-1 / 2, s}^{+}+\omega_{1, s}^{+} \Delta E_{i+1 / 2, s}^{+}-\omega_{0, s}^{-} \Delta E_{i+1 / 2, s}^{-}-\omega_{1, s}^{-} \Delta E_{i+3 / 2, s}^{-}\right],
$$

where

$$
\begin{aligned}
& \Delta E_{i+1 / 2, s}^{ \pm}=l_{s} \cdot \Delta E_{i+1 / 2}^{ \pm}, \\
& \Delta E_{i+1 / 2}^{+}=\Gamma_{i+1 / 2}^{-1} E_{i+1}-\tilde{E}_{i+1 / 2}^{L}, \\
& \Delta E_{i+1 / 2}^{-}=\tilde{E}_{i+1 / 2}^{L}-\Gamma_{i+1 / 2}^{-1} E_{i} .
\end{aligned}
$$

In the preceding equations $r_{s}$ (column vector) and $l_{s}$ (row vector) are the sth right and left eigenvectors of the Jacobian matrices, and they are evaluated using Roe's averages. The weights $\omega^{ \pm}$are defined as

$$
\begin{array}{ll}
\omega_{k, s}^{ \pm}=\frac{\alpha_{k, s}^{ \pm}}{\alpha_{0, s}^{ \pm}+\alpha_{1, s}^{ \pm}}, & (k=0,1), \\
\alpha_{0, s}^{+}=\frac{1}{3}\left(\varepsilon+I S_{0, s}^{+}\right)^{-2}, & \alpha_{1, s}^{+}=\frac{2}{3}\left(\varepsilon+I S_{1, s}^{+}\right)^{-2}, \\
\alpha_{0, s}^{-}=\frac{2}{3}\left(\varepsilon+I S_{0, s}^{-}\right)^{-2}, & \alpha_{1, s}^{-}=\frac{1}{3}\left(\varepsilon+I S_{1, s}^{-}\right)^{-2} .
\end{array}
$$

Here $\epsilon=10^{-7}$, and the smoothness indicators $I S_{k, s}, k=0,1$ were given in Ref. [11].

\subsubsection{P-WENO3 scheme}

Similar to the construction of P-WENO2 scheme, the P-WENO3 numerical flux is split to first-order and high-order parts. The first-order part for P-WENO3 scheme is the same as that for P-WENO2 scheme,

$$
\tilde{E}_{i+1 / 2}^{\mathrm{P}-\mathrm{WENO}}=\Gamma_{i+1 / 2}\left(\tilde{E}_{i+1 / 2}^{L}+\tilde{E}_{i+1 / 2}^{H 3}\right)=\tilde{E}_{i+1 / 2}^{\mathrm{P}-\mathrm{Roe}}+\Gamma_{i+1 / 2} \tilde{E}_{i+1 / 2}^{H 3} .
$$

The high-order flux of P-WENO3 scheme is defined as

$$
\tilde{E}_{i+1 / 2, s}^{H 3}=\frac{1}{6}\left[\omega_{0, s}^{+} \bar{q}_{0}^{+}+\omega_{1, s}^{+} \bar{q}_{1}^{+}+\omega_{2, s}^{+} \bar{q}_{2}^{+}+\omega_{0, s}^{-} \bar{q}_{0}^{-}+\omega_{1, s}^{-} \bar{q}_{1}^{-}+\omega_{2, s}^{-} \bar{q}_{2}^{-}\right],
$$

where

$$
\begin{array}{ll}
\bar{q}_{0}^{+}=-2 \Delta E_{i-3 / 2, s}^{+}+5 \Delta E_{i-1 / 2, s}^{+}, & \bar{q}_{1}^{+}=\Delta E_{i-1 / 2, s}^{+}+2 \Delta E_{i+1 / 2, s}^{+}, \\
\bar{q}_{2}^{+}=4 \Delta E_{i+1 / 2, s}^{+}-\Delta E_{i+3 / 2, s}^{+}, & \bar{q}_{0}^{-}=\Delta E_{i-1 / 2, s}^{-}-4 \Delta E_{i+1 / 2, s}^{-}, \\
\bar{q}_{1}^{-}=-2 \Delta E_{i+1 / 2, s}^{-}-\Delta E_{i+3 / 2, s}^{-}, & \bar{q}_{2}^{-}=-5 \Delta E_{i+3 / 2, s}^{-}+2 \Delta E_{i+5 / 2, s}^{-} .
\end{array}
$$

The definition of $\Delta E_{i+1 / 2, s}^{ \pm}$in the preceding equations is same as the P-WENO2 scheme. The weights $\omega^{ \pm}$are defined as 


$$
\begin{aligned}
& \omega_{k, s}^{ \pm}=\frac{\alpha_{k, s}^{ \pm}}{\alpha_{0, s}^{ \pm}+\alpha_{1, s}^{ \pm}+\alpha_{2, s}^{ \pm}}, \\
& \alpha_{0, s}^{+}=\frac{1}{10}\left(\varepsilon+I S_{0, s}^{+}\right)^{-2}, \quad \alpha_{1, s}^{+}=\frac{6}{10}\left(\varepsilon+I S_{1, s}^{+}\right)^{-2}, \\
& \alpha_{0, s}^{-}=\frac{3}{10}\left(\varepsilon+I S_{0, s}^{-}\right)^{-2}, \quad \alpha_{2, s}^{+}=\frac{3}{10}\left(\varepsilon+I S_{2, s}^{+}\right)^{-2},
\end{aligned}
$$

The smoothness indicators $I S_{k s}^{ \pm}, k=0,1,2$ can be found in Ref. [11]. In regions of smooth flow, the linear combination of the $\bar{q}_{k}^{ \pm}(k=0,1,2)$ given in Eq. (20) should reduce to the central scheme and give the optimal convergence properties possible [17]. These weights are thus known in the literature as the ideal weights or linear weights. These weights are given by

$$
\begin{array}{lll}
\alpha_{0, s}^{+}=\frac{1}{10}, & \alpha_{1, s}^{+}=\frac{6}{10}, & \alpha_{2, s}^{+}=\frac{3}{10}, \\
\alpha_{0, s}^{-}=\frac{3}{10}, & \alpha_{1, s}^{-}=\frac{6}{10}, & \alpha_{2, s}^{-}=\frac{1}{10} .
\end{array}
$$

\subsection{Time discretization}

The unfactored implicit equation is obtained by linearizing the flux vectors about the preceding time and dropping terms of second and higher order:

$$
\left[I+\Delta t\left(\delta_{\xi}^{-} \hat{A}_{\Gamma}^{+}+\delta_{\xi}^{+} \hat{A}_{\Gamma}^{-}+\delta_{\eta}^{-} \hat{B}_{\Gamma}^{+}+\delta_{\eta}^{+} \hat{B}_{\Gamma}^{-}+\delta_{\zeta}^{-} \hat{C}_{\Gamma}^{+}+\delta_{\zeta}^{+} \hat{C}_{\Gamma}^{-}\right)-\Delta t \hat{D}\right]^{n} \Delta \hat{Q}_{p, i, j, k}^{n}=\Delta t\left[\Gamma_{i, j, k}^{-1}(R H S)\right]^{n},
$$

where $(R H S)$ represents the right-hand-side of Eq. (13), I is the identity matrix, $\Delta Q_{p}^{n}=Q_{p}^{n+1}-Q_{p}^{n}$ is the increment of primitive variables, $\delta_{\xi}^{-}, \delta_{\eta}^{-}$and $\delta_{\zeta}^{-}$are backward difference operators and $\delta_{\xi}^{+}, \delta_{\eta}^{+}$and $\delta_{\zeta}^{+}$are forward difference operators in $\xi$, $\eta$ and $\zeta$ directions, respectively, and $\hat{A}_{\Gamma}^{ \pm}, \hat{B}_{\Gamma}^{ \pm}$and $\hat{C}_{\Gamma}^{ \pm}$are splitted Jacobian matrices of inviscid fluxes. The matrices are constructed so that the eigenvalues of '+' matrices are non-negative and those of '-' matrices are non-positive, that is, for example the matrices in $\xi$ direction $\hat{A}_{\Gamma}^{ \pm}$are obtained by

$$
\hat{A}_{\Gamma}^{ \pm}=R_{\xi}\left(\hat{\Lambda}_{\xi}^{ \pm} \pm v_{\xi} I\right) L_{\xi}^{ \pm},
$$

where $R_{\xi}$ and $L_{\xi}$ are similarity transformation matrices of the left and right eigenvectors of $\hat{A}_{\Gamma}$, and $\hat{\Lambda}_{\xi}^{ \pm}=\left(\hat{\Lambda}_{\xi} \pm\left|\hat{\Lambda}_{\xi}\right|\right) / 2$, where $\hat{\Lambda}_{\xi}$ is the diagonal matrix of eigenvalue of $\hat{A}_{\Gamma}$. The viscous corrected term $v_{\xi}$ is defined as [18]

$$
v_{\xi}=2\left(\frac{\mu_{\max }}{\rho}\right) \frac{|\nabla \xi|^{2} / J}{R e_{\infty}},
$$

where $\mu_{\max }$ must take the maximum value of diffusive coefficients in the system [19].

The stiffness caused by the turbulent production term can be reduced by treating the turbulent source functions implicitly. But the Jacobian of production term maybe positive, especially in the turbulent core region $\left(y^{+}>30\right)$, can be destabilizing the implicit system [20]. To compensate the destability, the Jacobian of the source term $\hat{D}=\partial \hat{H}_{6} / \partial \widetilde{v}$ which was evaluated by the pseudolinearization method proposed by Spalart and Allmaras [13] is used in the present study.

The LU-SGS implicit factorization scheme of Yoon and Kwak [21] for Eq. (21) can be derived by combining the advantages of LU factorization and SGS relaxation. The LU-SGS scheme can be written as

$$
(L+D) D^{-1}(D+U) \Delta \hat{Q}_{p}=-\Delta t \Gamma_{i, j, k}^{-1}(R H S)^{n} .
$$

The operators $L, U$ and $D$ are detailedly described in Ref. [3] and Eq. (22) can be solved in three steps [3,21].

\subsection{Boundary condition}

The mean flow and turbulent transport equations presented in preceding sections represent an initial-boundary-value problem. To solve these equations, it is necessary to impose initial and boundary conditions. A uniform flowfield is chosen as the initial conditions for the mean flow equations. A uniform value of $\widetilde{v}=O(1)$ is set as the initial guess.

The boundary conditions of the mean flow are set as follows: (1) No-slip boundary conditions for velocities are adopted on the solid surface, which is assumed to be an adiabatic wall. (2) The density and pressure on the wall are set to be equal to the values of the node points next to the wall. This gives first-order accuracy at the wall. (3) In the far field, a locally onedimensional characteristic type of boundary condition is used. The flow values on far-field boundary $Q_{p, b}$ can be evaluated by

$$
Q_{p, b}=\frac{1}{2}\left(Q_{p, \infty}+Q_{p, I}\right)-\frac{1}{2} \operatorname{sgn}\left(A_{\Gamma}\right)\left(Q_{p, \infty}-Q_{p, I}\right)
$$

where $Q_{p, \infty}$ is the values of primitive variables on freestream status, $Q_{p, I}$ is the values on the nearest inner point, $\operatorname{sgn}\left(A_{\Gamma}\right)=R \operatorname{sgn}(\Lambda) L$, and $\operatorname{sgn}(\Lambda)$ is a diagonal matrix, whose elements are +1 or -1 for positive or negative eigenvalue of matrix $A_{\Gamma}$, respectively. For the turbulent transport equation, a zeroth-order extrapolation is used to specify conditions at the far field. The value of $\widetilde{v}$ is set to zero at solid wall. 


\section{Results and discussions}

\section{1. $2 D$ driven cavity flow}

The first testing problem is the lid driven squared cavity flow with domain length 1 . A $65 \times 65$ non-uniform grid is used and the grid points are exponentially stretched away from the wall and the minimum grid spacing near the wall is 0.005 . Results were obtained for Reynolds number of 3200 which is based on lid velocity and length of cavity, under an isothermal condition and a Mach number of 0.001 for the moving lid. Three numerical schemes, P-STVD, P-WENO2, P-WENO3 are employed. Fig. 1(a)-(c) shows the results of streamline contours calculated by the three different schemes. The streamline plots show a large primary vortex near the center of cavity, along with two secondary vortices at the bottom corners and one secondary vortex near the upper-left corner. The size of the secondary vortex the bottom corners by P-STVD scheme is smaller than that by P-WENO2 and P-WENO3 schemes. Also, the secondary vortex at the upper-left corner by P-STVD scheme is not present, but obviously exists by P-WENO2 and P-WENO3 schemes. To verify the accuracy of numerical solutions, Fig. 2(a) and (b) depicts, respectively, the comparison of $u$ velocity component along the vertical centerline and $v$ velocity component
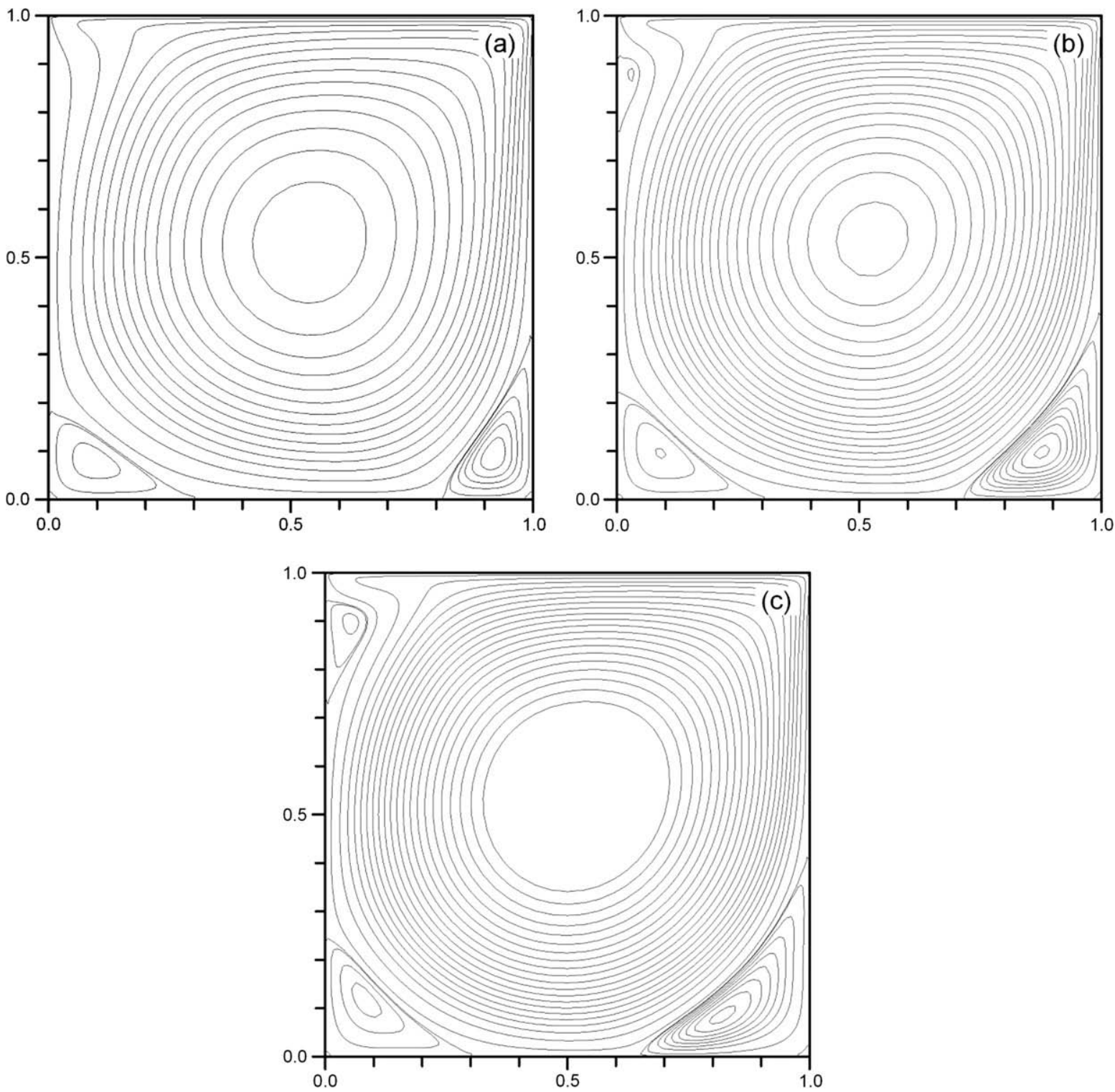

Fig. 1. Streamline contours for flow in a driven cavity for $R e_{\infty}=3200$ by (a) P-STVD, (b) P-WENO2, (c) P-WENO3 scheme. (The contourlines with min. -0.0034 , max. 0.1 and increment 0.0004 for negative values and 0.005 for positive values.) 

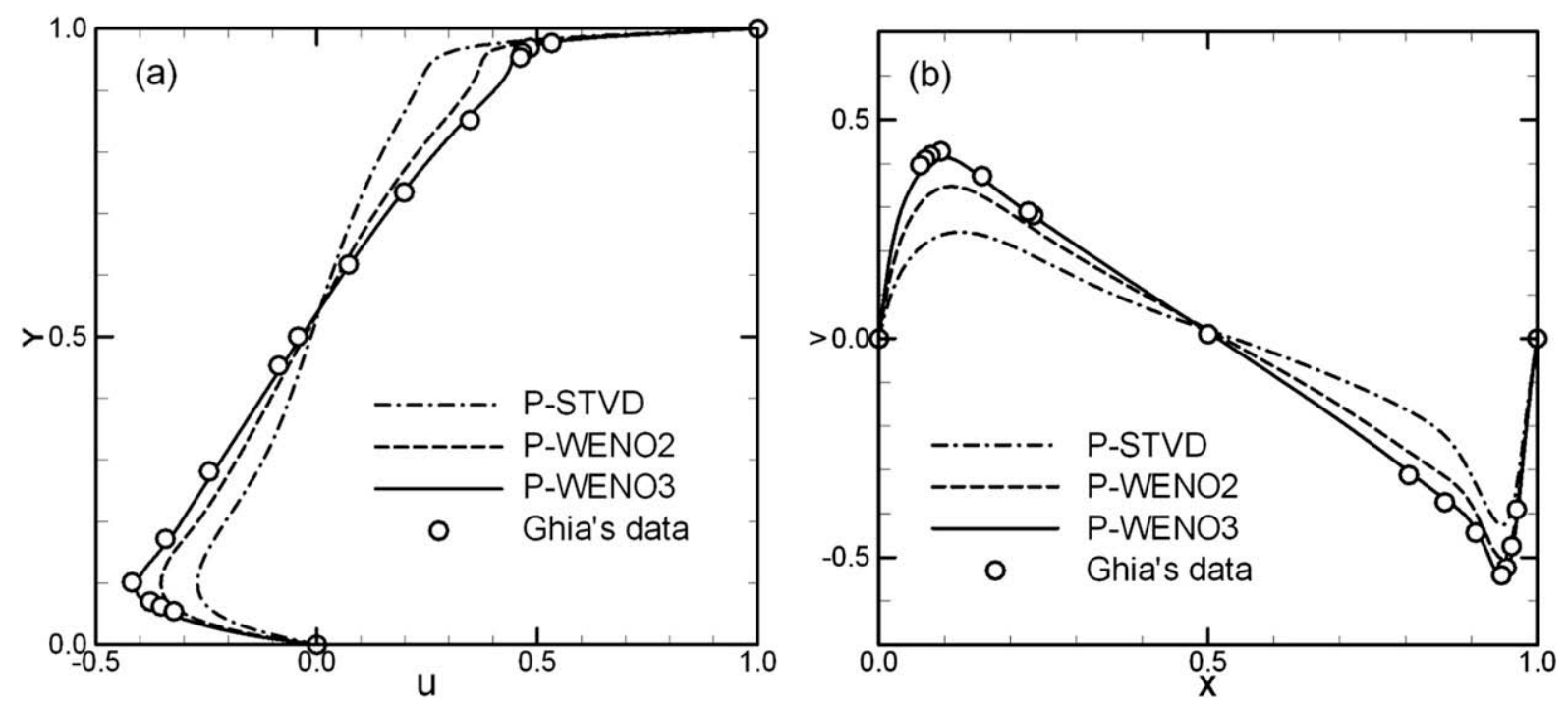

Fig. 2. Comparison of (a) $u$ velocity along the vertical centerline, and (b) $v$ velocity along the horizontal centerline for the driven cavity at $R e_{\infty}=3200$

along the horizontal centerline with numerical solutions by Ghia et al. [22] using a fine $129 \times 129$ uniform grid. Excellent agreement can be observed for the results of P-WENO3 scheme even with the current coarse grid.

Fig. 3 shows the comparison of convergence histories among P-Roe, P-STVD, P-WENO2 and P-WENO3 schemes. In these cases, the CFL number is fixed to 30. A reasonably good convergence rate to steady-state solution can be obtained. For P-Roe scheme, it requires about 1600 iterations for seven orders of $L_{2}$ norm convergence. And it requires 4000 and 6400 iterations for P-STVD and P-WENO2 scheme, respectively. The convergence rate is related to the damping of numerical scheme. The high-order schemes, P-WENO2 and P-WENO3, converge relatively slower than P-Roe or P-STVD schemes. The convergence histories of high-order schemes contain a flowfield evolution process, which generated by vortices interaction. It is suspected that the process is due to the strong transient nature of the flow where several significant secondary vortices appear and interact with the main circulating vortex. The superior performance of P-WENO scheme to the P-STVD scheme can be clearly seen for this flow that is characteristized by multiple vortices.

The computational costs per time step for 2D cavity flow with P-STVD, P-WENO2 and P-WENO3 scheme are $0.079,0.091$ and $0.093 \mathrm{~s}$, respectively. With the same cases, but using non-preconditioned STVD, WENO2 and WENO3 schemes, the computational costs per time step are $0.049,0.054$ and $0.056 \mathrm{~s}$, which are around $60 \%$ of that of preconditioned schemes. The computational costs per time step with P-WENO2 and P-WENO3 schemes are around 1.15 and 1.17 times of that of P-STVD

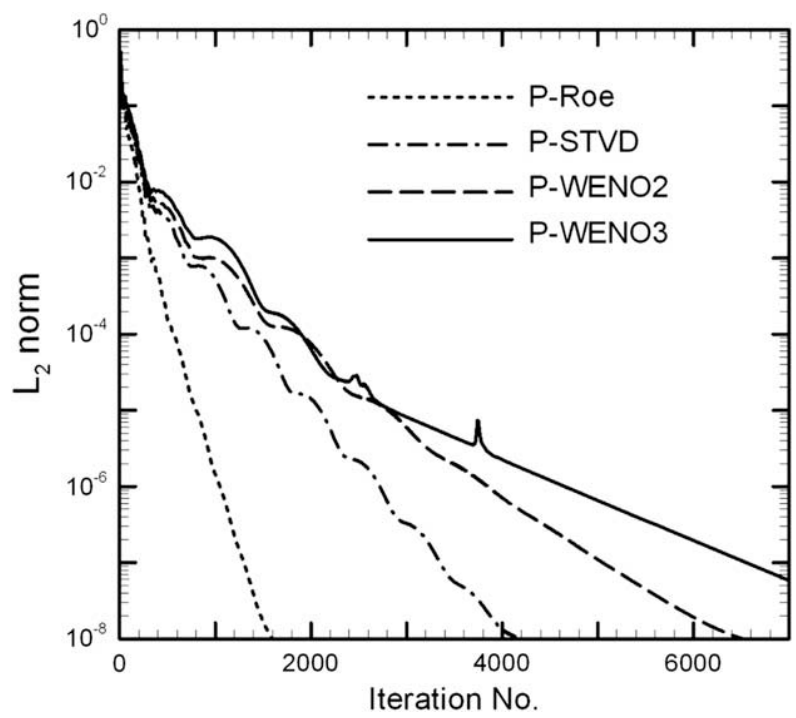

Fig. 3. Convergence history for cavity flow at $R e_{\infty}=3200$. 
scheme, respectively. With maximum CFL number 30, the time steps for reaching seven orders of convergence with PWENO2 and P-WENO3 schemes are around 1.5 and 2.0 times to the P-STVD scheme, respectively. Therefore the total computing time are 1.73 and 2.34 times more for the P-WENO2 and P-WENO3 schemes, respectively.

Fig. 4(a) and (b) depicts, respectively, the comparison of $u$ velocity component along the vertical centerline, and $v$ velocity component along the horizontal centerline between nonlinear and linear weights P-WENO3 scheme. Fig. 5 shows the comparison of convergence history. It is found that the results of nonlinear weights agree very well with the linear weights for the case of low speed flow. Obviously, the usage of the linear weights will reduce the computational cost. But the computational cost depends on several modules of the code, for examples, the implicit module would be the major consumer of CPU time. In current computations, the overall cost of the computation by linear weights was reduced by about $5 \%$ compared with that nonlinear weights.

\subsection{Low subsonic viscous flow over S809 airfoil}

The second problem considers viscous flow over airfoil at moderate angle of attack. The S809 airfoil was designed specially for horizontal-axis-wind-turbine (HAWT) applications. The thickness ratio of the airfoil is $21 \%$. A $600 \mathrm{~mm}$ chord length model of the $\$ 809$ airfoil has been tested in a $1.8 \mathrm{~m} \times 1.25 \mathrm{~m}$ low turbulence wind tunnel [22]. Four grid systems of C-type topology are generated for grid-independency testing. The grid points are $185 \times 49,273 \times 73,409 \times 109$ and $601 \times 145$ from coarse to fine grid system, and there are 137, 207, 321 and 481 points on airfoil surface, respectively, and the outer boundary for all four grid systems is about 20 chord lengths away from airfoil. The grid points in the direction normal to the airfoil surface are exponentially stretched away from the airfoil surface with a minimum grid spacing $10^{-5}$ chord length at airfoil surface. Grid points are clustered at the leading and trailing edges with a spacing of 0.0004 and 0.0015 chord length, respectively. A close-up view of the coarsest grid system is shown in Fig. 6 . The freestream Mach number is 0.001 , Reynolds number is $2 \times 10^{6}$ and angle of attack is $9.22^{\circ}$. The computational results of lift and drag coefficients for various computational grid systems with P-STVD, P-WENO2 and P-WENO3 schemes, and along with the experimental data by Somers [23] are listed in the following table:

\begin{tabular}{|c|c|c|c|c|c|c|}
\hline \multirow[t]{2}{*}{ Grid } & \multicolumn{2}{|l|}{ P-STVD } & \multicolumn{2}{|c|}{ P-WENO2 } & \multicolumn{2}{|c|}{ P-WENO3 } \\
\hline & $C_{L}$ & $C_{D}$ & $C_{L}$ & $C_{D}$ & $C_{L}$ & $C_{D}$ \\
\hline $185 \times 49$ & 1.0651 & 0.03793 & 1.0856 & 0.02373 & 1.0742 & 0.02054 \\
\hline $273 \times 73$ & 1.0758 & 0.02812 & 1.0870 & 0.02123 & 1.0829 & 0.02008 \\
\hline $409 \times 109$ & 1.0819 & 0.02373 & 1.0892 & 0.02036 & 1.0843 & 0.02005 \\
\hline $601 \times 145$ & 1.0838 & 0.02213 & 1.0893 & 0.02015 & 1.0846 & 0.02005 \\
\hline \multicolumn{3}{|c|}{ Exp. data by Somers [23] } & \multicolumn{2}{|c|}{$C_{L}=1.0385$} & \multicolumn{2}{|c|}{$C_{D}=0.0214$} \\
\hline
\end{tabular}
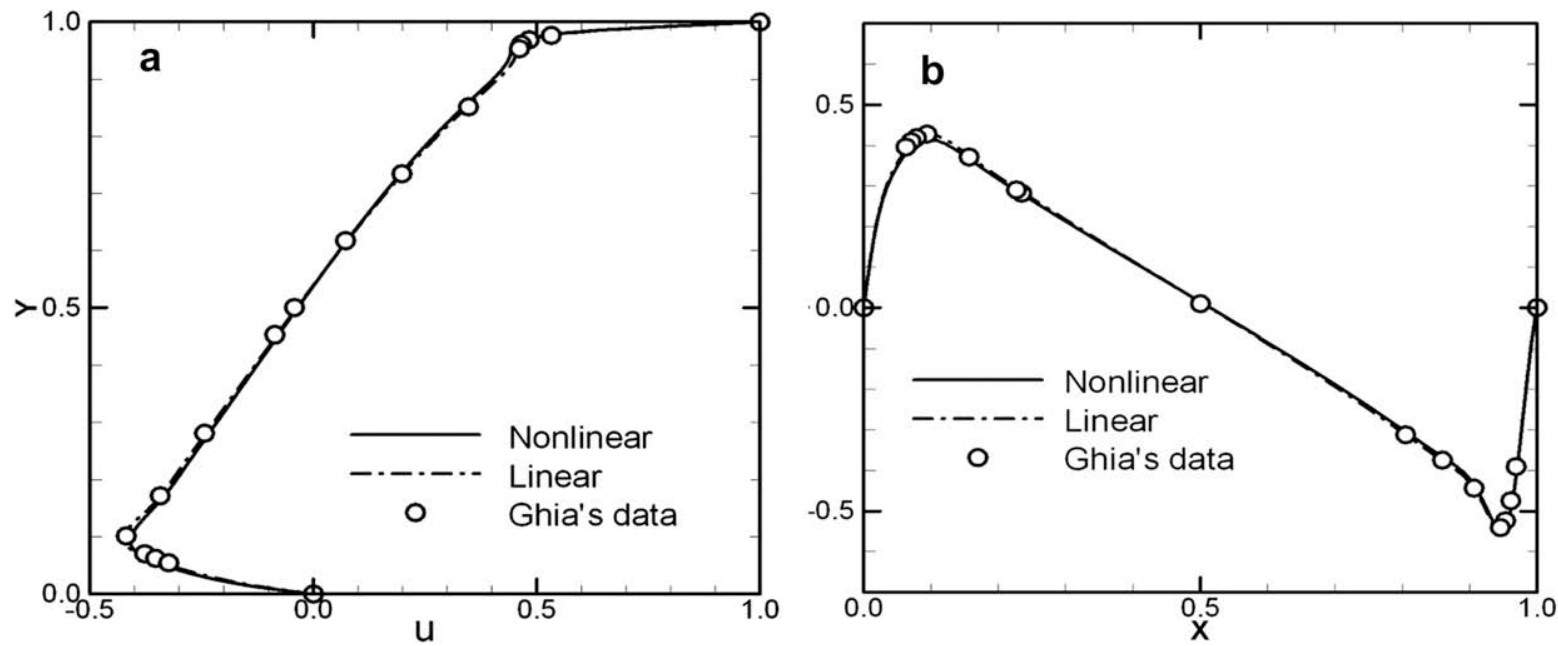

Fig. 4. Comparison of (a) $u$ velocity along the vertical centerline, and (b) $v$ velocity along the horizontal centerline for the driven cavity at $R e_{\infty}=3200$ between linear weights and nonlinear weights P-WENO3 scheme. 


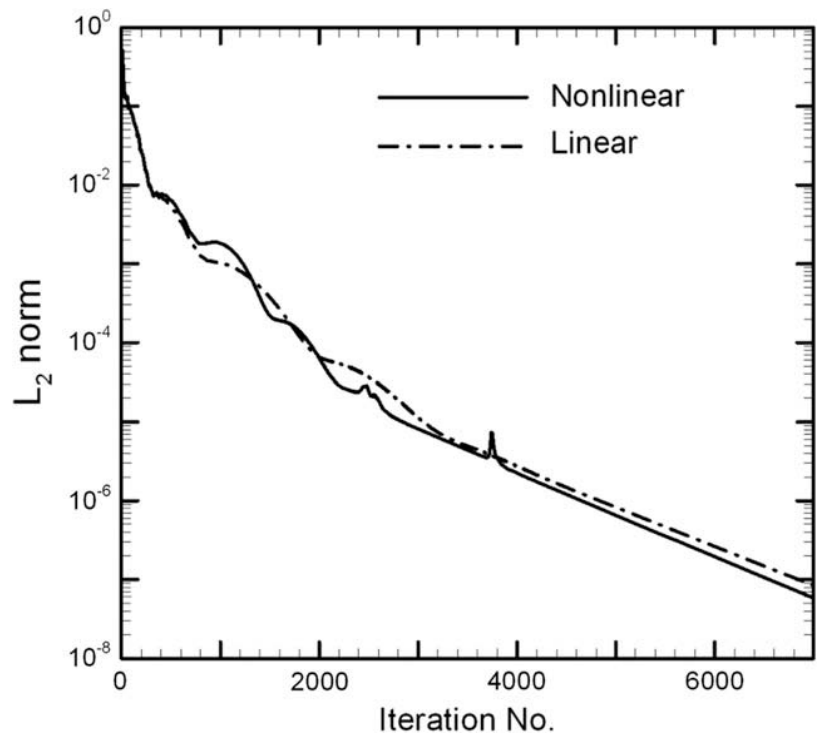

Fig. 5. Comparison of Convergence history for cavity flow at $R e_{\infty}=3200$ between linear weights and nonlinear weights.

As shown in the table, the lift coefficients computed by all twelve cases agree with one another, and are slightly higher than the experimental data. The discrepancies in lift coefficients by all three schemes over various grid systems are under $5 \%$. The drag coefficients computed by P-STVD scheme are obviously dependent on grid systems. It can be seen that only the finest grid system $(601 \times 145)$ attains accurate results. In contrast, the drag coefficients computed by P-WENO3 scheme with various grid systems agree very closely with one another and are slightly lower than the experiment data being $5 \%$ in difference. The drag coefficients computed by P-WENO2 scheme are also in good agreement except for results using the coarsest grid system $(185 \times 49)$. This is readily discerned by looking at the table. The sensitivity study among the grid systems demonstrated that the $273 \times 73$ grid and finer grids can give reasonable results by using P-WENO2 or P-WENO3 schemes.

The distributions of pressure coefficients obtained by P-STVD and P-WENO3 schemes with various grid systems are presented in Fig. 7(a) and (b), respectively, along with the experimental data [23]. As can be seen, the distributions agree reasonably well with the experimental data except slight differences in the upper surface near the trailing edge region in which separation occured. The results of the P-WENO3 scheme with various grid systems are almost the same, but for the results of the P-STVD scheme, small deviations in the upper surface near the leading edge are caused by the effects of using different grid systems. This deviations are depicted in Fig. 8 which shows the close-up of leading edge region. Excellent agreement can be observed for the P-WENO3 results even with the $185 \times 49$ coarse grid system.

The convergence histories of all three schemes with $185 \times 49$ and $601 \times 145$ grid systems are shown in Fig. 9(a) and (b). All three schemes have similar convergence history. After the residuals have decayed for two orders of magnitude in 1000 iterations, the rate of convergence is slowing down. The preconditioned system requires about 4500 iterations to reduce the $L_{2}$ norm by four orders of magnitude.

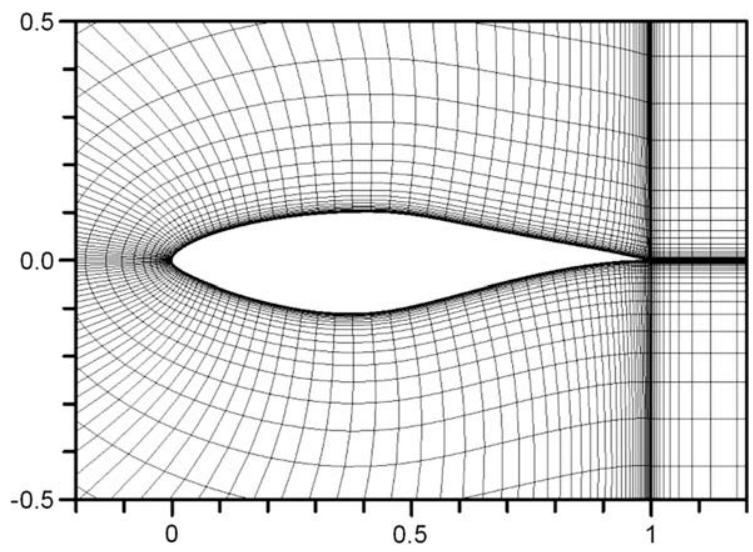

Fig. 6. A close-up view of $185 \times 49$ grid system for $\$ 809$ airfoil. 

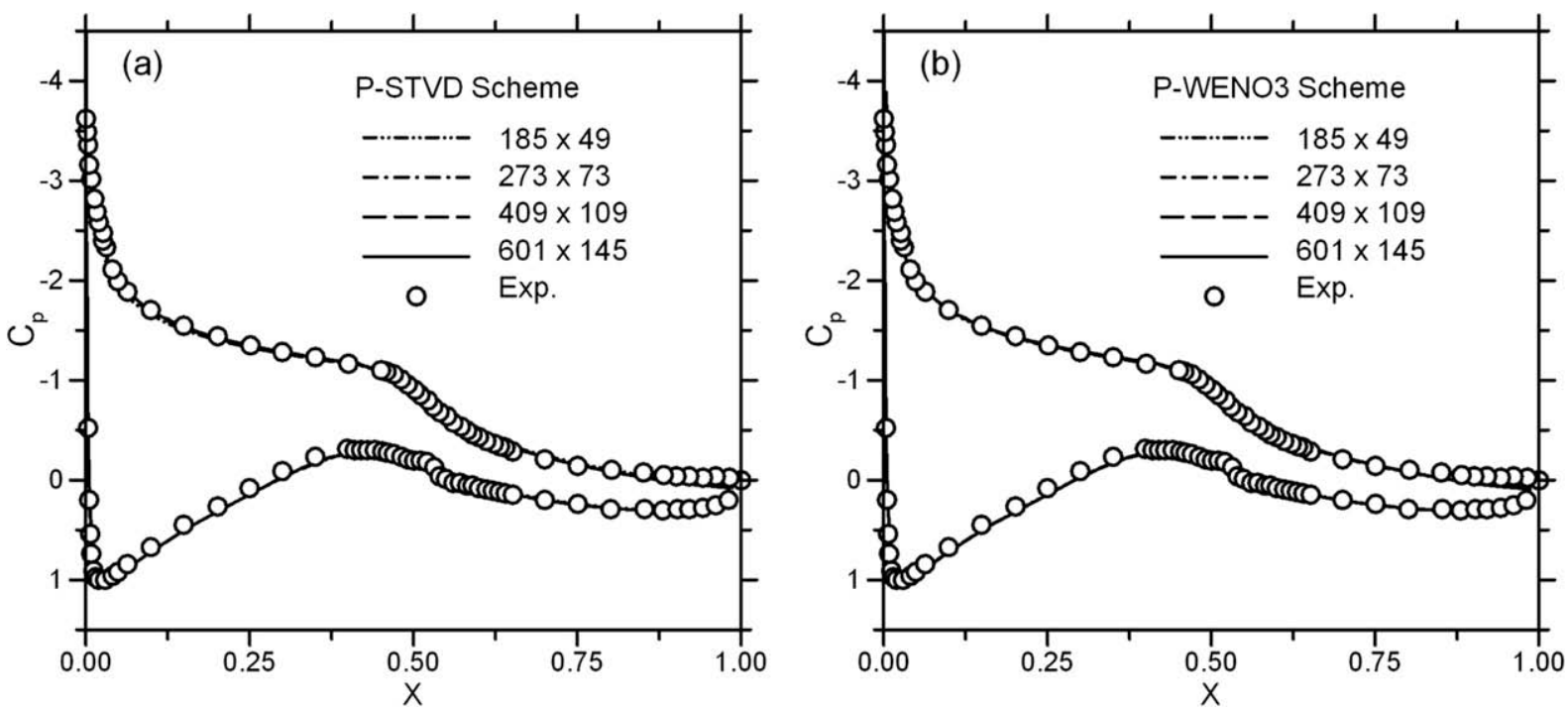

Fig. 7. S809 airfoil surface pressure distribution at $M_{\infty}=0.001, \alpha=9.22^{\circ}, R e_{\infty}=2 \times 10^{6}$; comparison of different grid systems by (a) P-STVD scheme and (b) P-WENO3 scheme and along with experimental data.

\subsection{D low speed viscous flow over 6:1 prolate spheroid}

The flow past a prolate spheroid has been extensively studied and is a benchmark problem for studying three-dimensional separated flow structures. A multi-block point matched grid system, which composed of an $\mathrm{O}-\mathrm{O}$ type and an $\mathrm{H}-\mathrm{H}$ type grid topologies, is used for the computations. A representative $0-0$ type grid system of $57(\xi) \times 25(\eta) \times 57(\zeta)$ is shown in Fig. 10 for the bare prolate spheroid, where $\xi$ is in the streamwise direction, $\eta$ in the circumferential direction and $\zeta$ in the normal direction. The grid points are clustered in the normal direction near the body surface for resolving the thin viscous layers. The minimum grid spacing in the normal direction normalized with the major axis is taken to be $2 \times 10^{-5}$. To avoid the polar singularity, an $\mathrm{H}-\mathrm{H}$ type grid of $7 \times 13 \times 57$ was designed near the leading and trailing points and extended upstream and downstream as shown in Fig. 10. The outer boundary shape of the computational domain was elliptic, extending 25 major axes length in front of and in the downstream directions. To investigate the effects of grid refinements, calculations also performed on a coarse grid system with $29(\xi) \times 13(\eta) \times 29(\zeta)$ points, which was taken as every other point from the fine grid system. The freestream Mach number of 0.01 , Reynolds number of $1.6 \times 10^{6}$ and angle of attack of $10^{\circ}$ have

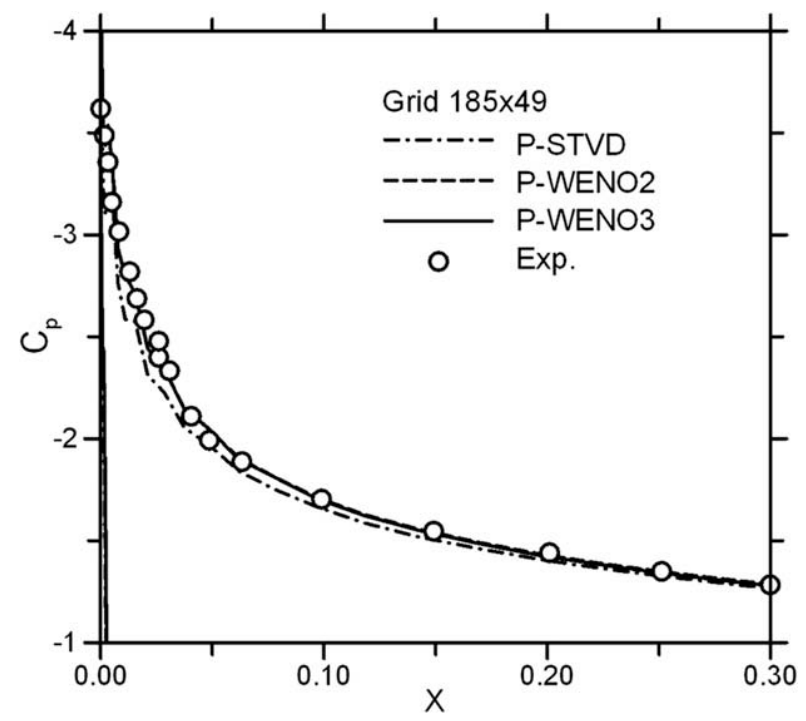

Fig. 8. S809 airfoil surface pressure distribution at $M_{\infty}=0.001, \alpha=9.22^{\circ}, R e_{\infty}=2 \times 10^{6}$; comparison of different schemes with $185 \times 49$ grid system (with experimental data and close-up of leading edge region). 

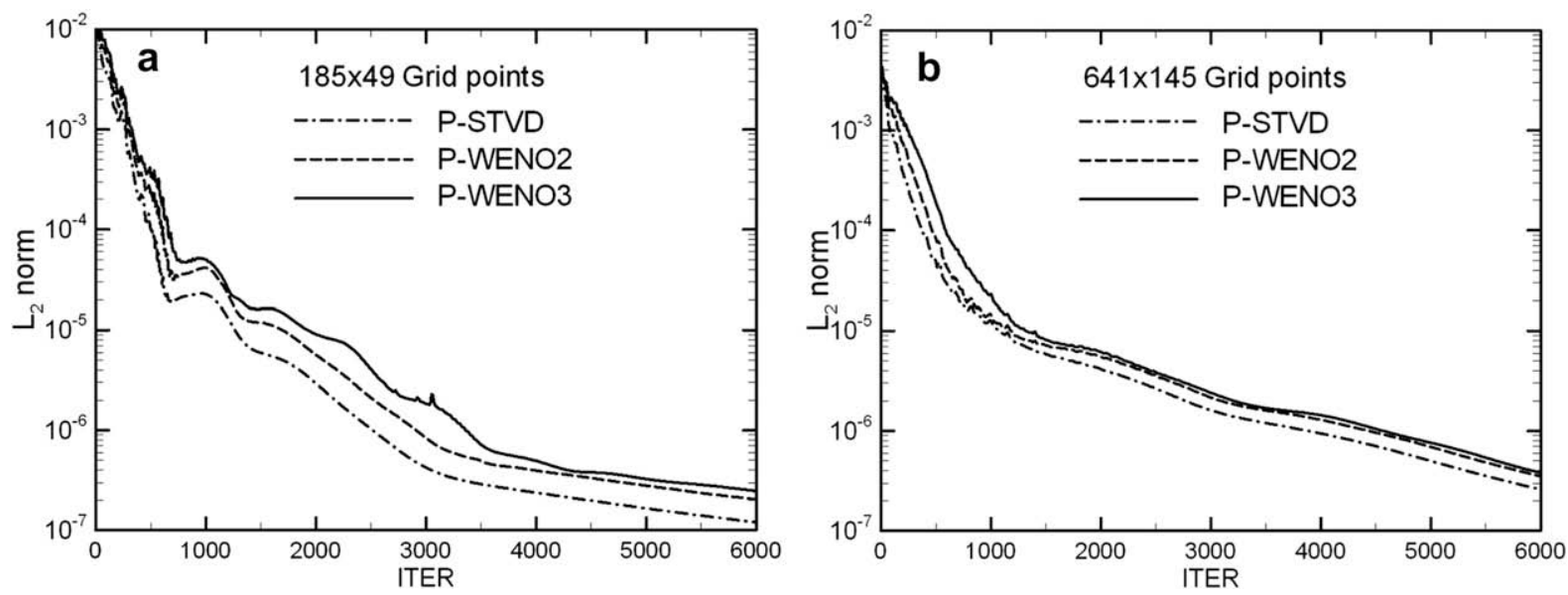

Fig. 9. Convergence history for S809 airfoil with (a) $185 \times 49$ (b) $601 \times 145$ grid systems at $M_{\infty}=0.001, \alpha=9.22^{\circ}, R_{\infty}=2 \times 10^{6}$.

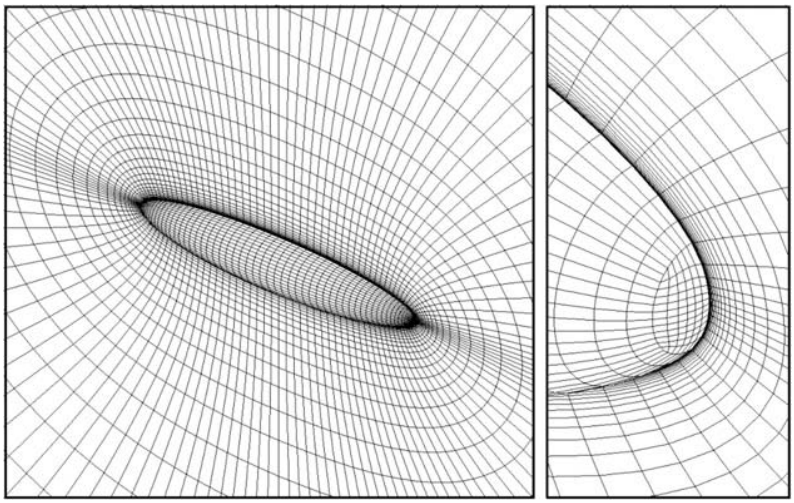

Fig. 10. A composite grid system of $\mathrm{O}-\mathrm{O}$ type and $\mathrm{H}-\mathrm{H}$ type grid topologies for 6:1 prolate spheroid.

been used as flow conditions. The computational results are obtained by P-STVD and P-WENO3 schemes with fine and coarse grid systems. The computed surface pressures and the experimental results by Meier and Cebeci [24] have been compared at symmetric plane on the prolate spheroid and presented in Fig. 11(a) and (b) for coarse and fine grid system. Fig. 12(a) and (b) depicts the close-up view of the leading edge region for the P-STVD and P-WENO3 schemes. The comparisons show a rea-
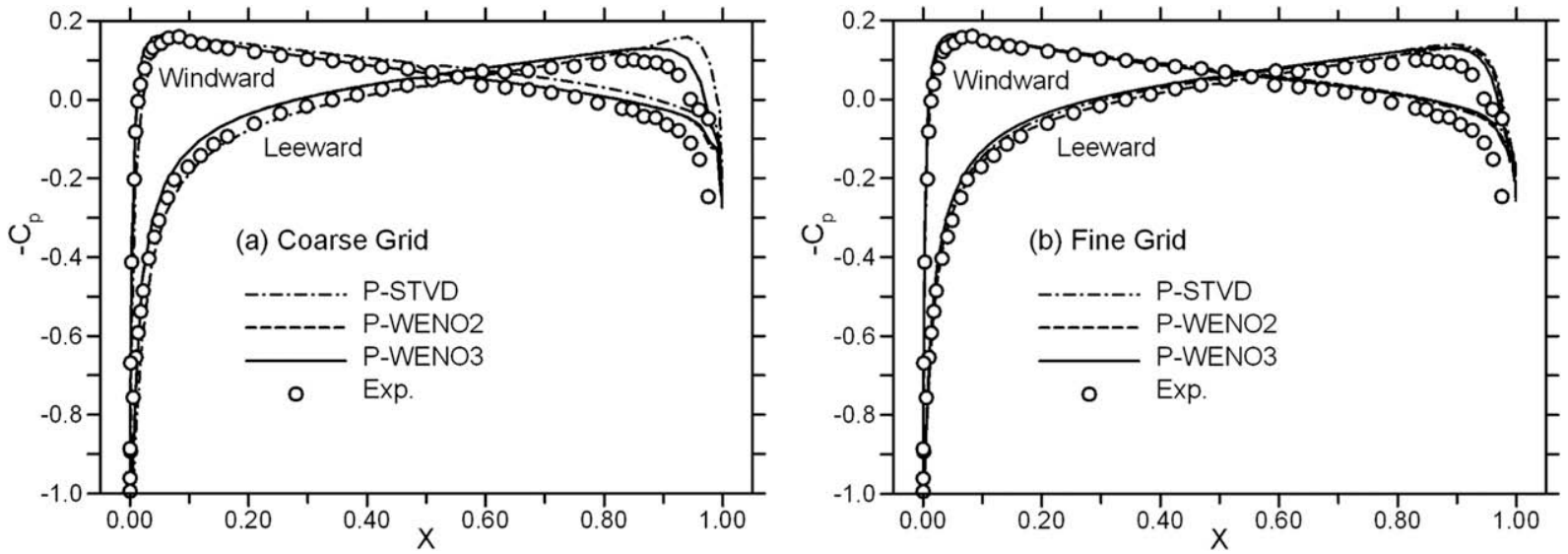

Fig. 11. Symmetric plane surface pressure distribution for $6: 1$ prolate spheroid at $M_{\infty}=0.01, \alpha=10^{\circ}, R e_{\infty}=1.6 \times 10^{6}$; comparison of various schemes with (a) coarse and (b) fine grid systems. 

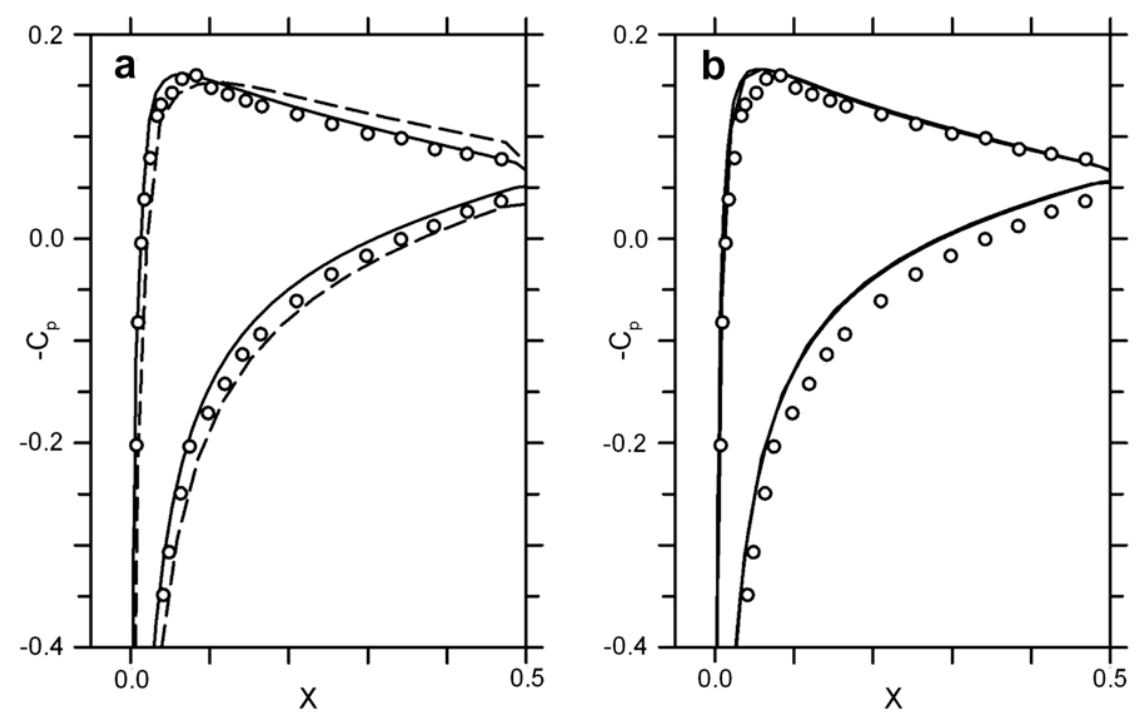

Fig. 12. Symmetric plane surface pressure distribution for $6: 1$ prolate spheroid at $M_{\infty}=0.01, \alpha=10^{\circ}, R e_{\infty}=1.6 \times 10^{6}$; comparison of different schemes by (a) P-STVD and (b) P-WENO3 scheme (close-up of leading edge region solid line: fine grid, dash line: coarse grid).

sonable agreement except for the region near the rear end where the pressure changes rapidly. The deviations near the rearstagnation points might be attributed to usage of one-equation turbulence model which is not accurate for the separation flow. It can also be seen that there are almost no differences in the pressure distributions between coarse and fine grid systems in the P-WENO3 scheme, but for the results of the P-STVD scheme, some obvious deviations occur. The effects of grid refinement make evident that accurate results are obtained by the P-WENO3 scheme even with the corresponding coarse grid system, but not by the P-STVD scheme.

The convergence histories for all three schemes with fine grid systems are shown in Fig. 13, where the averaged $L_{2}$-norm of residuals is plotted vs iteration number. It can be seen that faster convergence was obtained by P-STVD scheme, which requires about 2000 iterations for five orders of magnitude, but P-WENO3 scheme requires 4000 iterations.

\subsection{Transonic flow over ONERA-M6 wing}

Transonic turbulent flow over the three-dimensional ONERA-M6 wing has been chosen to verify the characteristics of the preconditioned system at transonic speed solved by WENO scheme. The ONERA M6 wing is a no-twist, semi-span wing with sweep angle of $30^{\circ}$. The airfoil section is an ONERA D symmetric section with 0.1 maximum thickness-to-chord ratio at $37.6 \%$ chord length from sectional leading edge. The wing is tapered with a taper ratio of 0.562 and has an aspect ratio of 3.8. Data for comparison consists of pressure coefficients at sections along the span of the wing obtained in the experiment performed by Schmitt and Charpin as reported in the AGARD report [25].

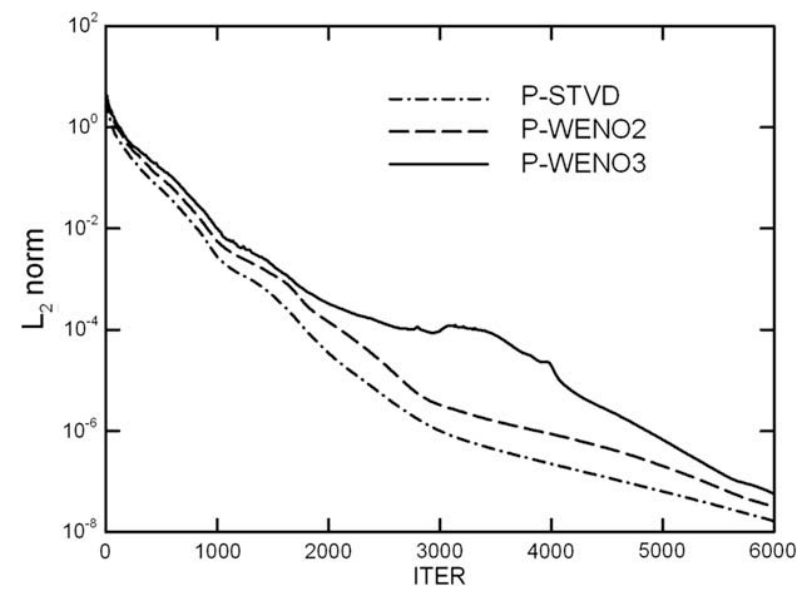

Fig. 13. Convergence history for flow over 6:1 prolate spheroid at $M_{\infty}=0.01, \alpha=10^{\circ}, R e_{\infty}=1.6 \times 10^{6}$, with fine grid system. 
An C-O type grid system containing $145 \times 49 \times 25$ grid points in streamwise, spanwise and body normal directions, respectively. The outer boundaries are extended to 20 chord lengths in all directions. The grid points in the normal direction are exponentially stretched away from the wing surface with the minimum grid spacing $3 \times 10^{-4}$ semi-span. The freestream Mach number of 0.84 , Reynolds number of $1.74 \times 10^{7}$ based on wing semi-span, and angle of attack of $3.06^{\circ}$ have been used as flow conditions. At these flow conditions, shock boundary interactions occur at upper and lower wing surface, and induce complex flow topology. The solutions were calculated using P-STVD, P-WENO2 and P-WENO3 schemes. The surface pressure distributions in Fig. 12 show the comparisons between experimental data [25] and computational results by all three schemes at various spanwise stations: $y / b=0.2,0.44,0.9,0.95$, where $b$ is the wing semi-span. Transonic flow over wing upper surface results in a double-shock flow topology near the wing root region, which is depicted in Fig. 14(a) and (b). However, in the region near the wing tip, the shocks coalesced to form one at the position of $25 \%$ chord length which are shown in Fig. 14(c) and (d), and the shocks are stronger than the previous ones. There are no apparent discrepancies in pressure distribution and shock-wave position occured between P-WENO2 and P-WENO3 schemes, and both results are in reasonable agreement with experimental data and are more accurate than those of P-STVD scheme in terms of both shock location and strength. Fig. 15(a) and (b) shows the pressure contours along the upper surface computed by P-STVD and P-WENO3 schemes. The surface flow obviously results in the lambda shock pattern for transonic flow on a swept wing. Clearly, the results of the P-WENO3 scheme are more refined. The convergence histories of the three different schemes are shown in Fig. 16. It can be seen that faster convergence was obtained by the P-STVD scheme, which requires about 5000 iterations for five orders of convergence, while for the P-WENO3 scheme, it requires 6000 iterations for almost four orders.

Fig. 17(a) and (b) shows the comparison of surface pressure distribution at span $y / b=0.2$ and $y / b=0.8$, respectively, between the results using P-WENO2 (with preconditioning) and WENO2 (without preconditioning) schemes based on same grid system. The results agree well with each other except for very small deviations near the shock on upper surface. The comparison of convergence history is shown in Fig. 18. The P-WENO2 scheme converges relatively slower than the WENO2 scheme.
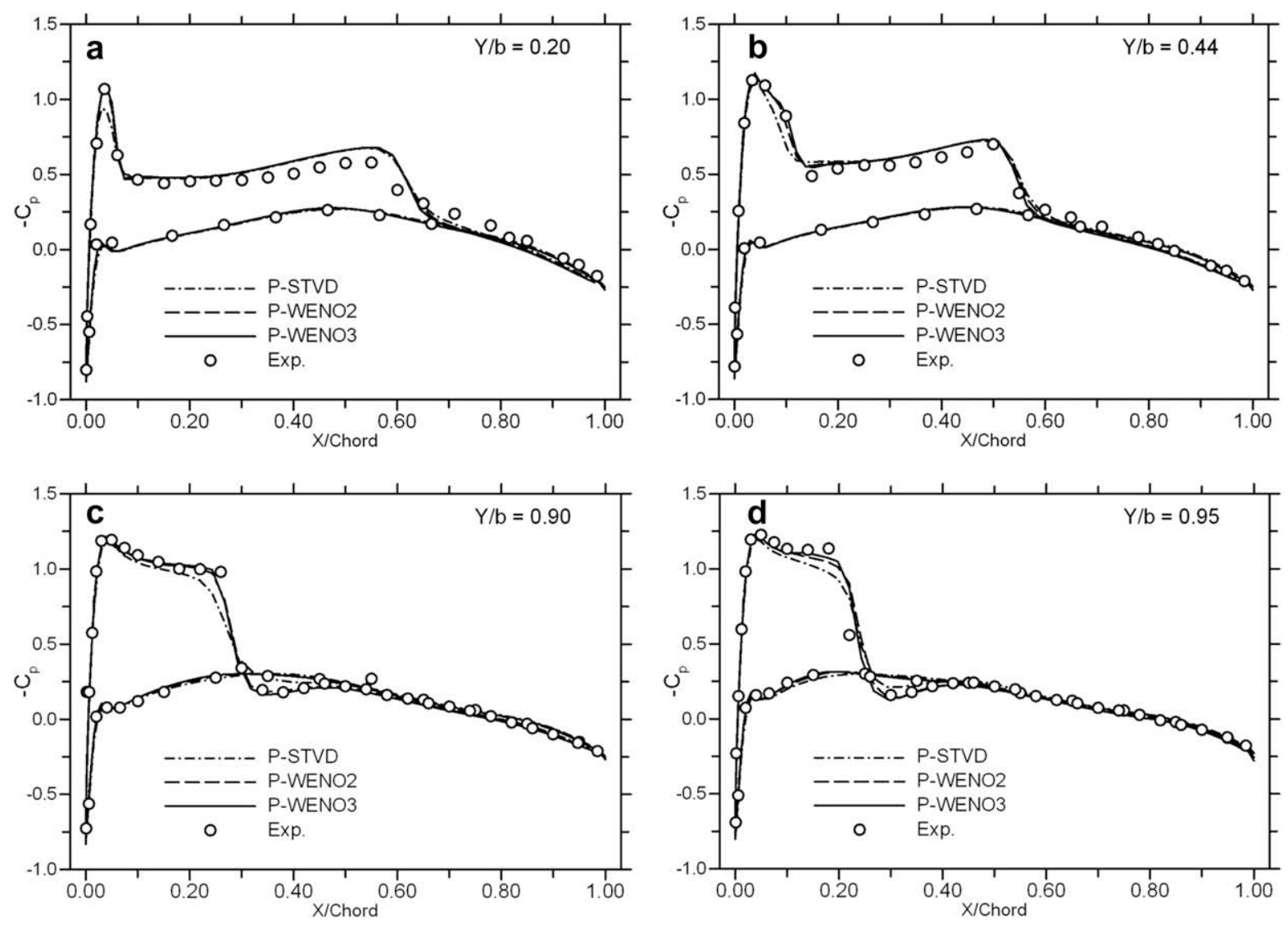

Fig. 14. Surface pressure distribution for ONERA M6 wing at $M_{\infty}=0.84, \alpha=3.06^{\circ}$ and $R e_{\infty}=1.74 \times 10^{7}$; comparison of schemes along spanwise chordline (a) $y / b=0.2$, (b) $y / b=0.44$, (c) $y / b=0.9$ and (d) $y / b=0.95$. 

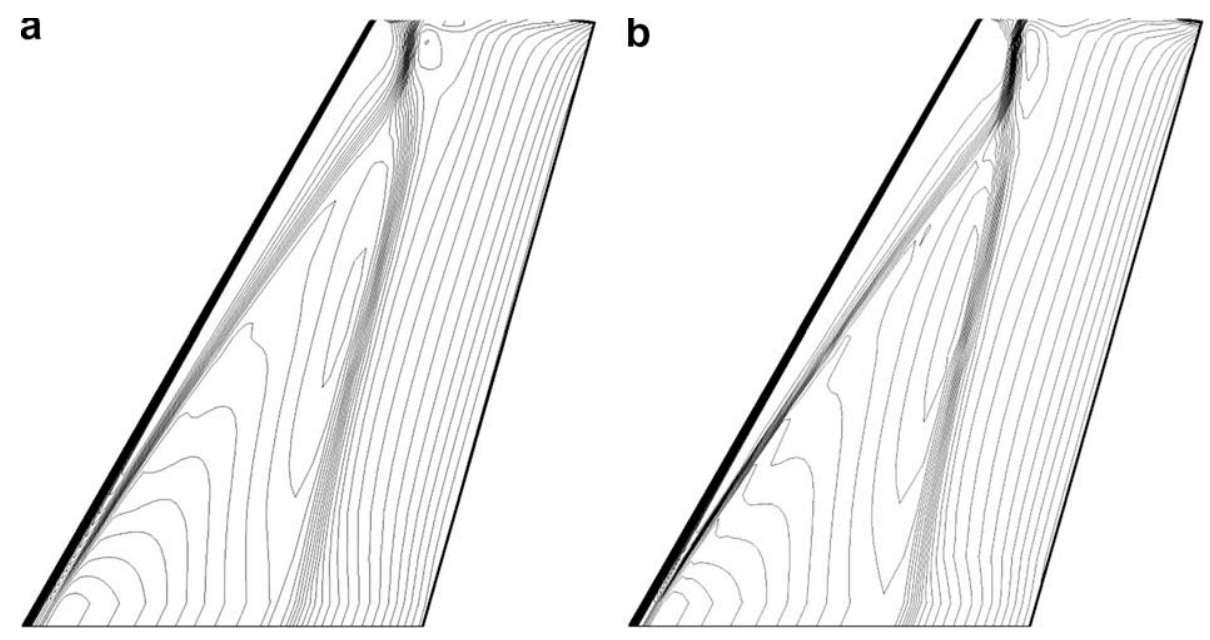

Fig. 15. Upper surface pressure contours for ONERA M6 wing at $M_{\infty}=0.8395, \alpha=3.06^{\circ}$ and $R e_{\infty}=1.74 \times 10^{7}$; comparison of schemes by (a) P-STVD and (b) P-WENO3.

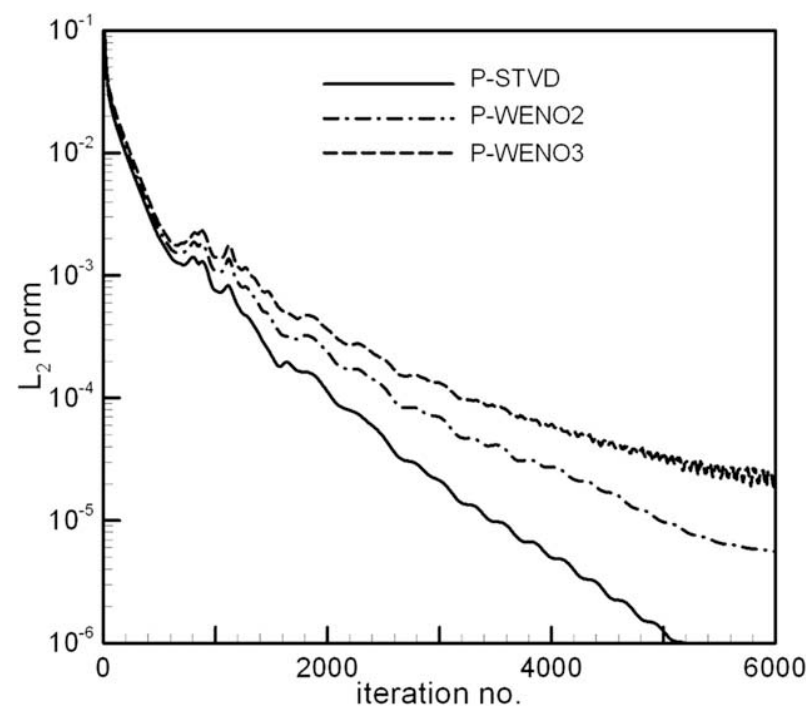

Fig. 16. Convergence history for ONERA M6 wing at $M_{\infty}=0.84, \alpha=3.06^{\circ}$ and $R e_{\infty}=1.74 \times 10^{7}$.

\subsection{Hypersonic flow over HB-2 model}

The hypervelocity ballistic-type model, designated HB-2, was designed in accordance with specifications originally suggested by the von Karman Gas Dynamics Facility (VKF) in 1959. The purpose of designing and testing this model was to extend the speed range for correlation of wind tunnel data, after the manner adopted by the AGARD many years ago for the transonic and supersonic regimes [26]. Until now, the HB-2 model has been widely used to evaluate aerodynamic test facilities and consequently, a large amount of experimental data is available.

The configuration of the HB-2 model is blunted cone-cylinder-flare geometry. The geometric parameters include the radius of blunted nose of $0.3 d$, where $d$ is the diameter of cylinder, the half cone angle of $25^{\circ}$, the cone length of $0.362 d$, the length of cylinder of $2.536 \mathrm{~d}$ and the flare angle of $10^{\circ}$. The non-dimensional total length of the model is $4.9 \mathrm{~d}$. Fig. 19 shows the composite grid system. A C-O type grid discretized most of the computational domain with 101 points in the streamwise direction, 49 points in the circumferential direction, and 65 in the normal direction. The grid points are clustered in the normal direction near the body surface for resolving the thin viscous layers. The minimum grid spacing in the normal direction is taken to be $3 \times 10^{-5}$. To avoid the polar singularity, an $\mathrm{H}-\mathrm{H}$ type grid of $13 \times 25 \times 65$ was designed near the blunted nose and extending to upstream.

The freestream Mach number of 5.1, Reynolds number of $2.32 \times 10^{6}$, and angle of attack of $15^{\circ}$ have been used as flow conditions. At these flow conditions, the flow structures consist of bow shock, cross flow separation and re-attachment, 

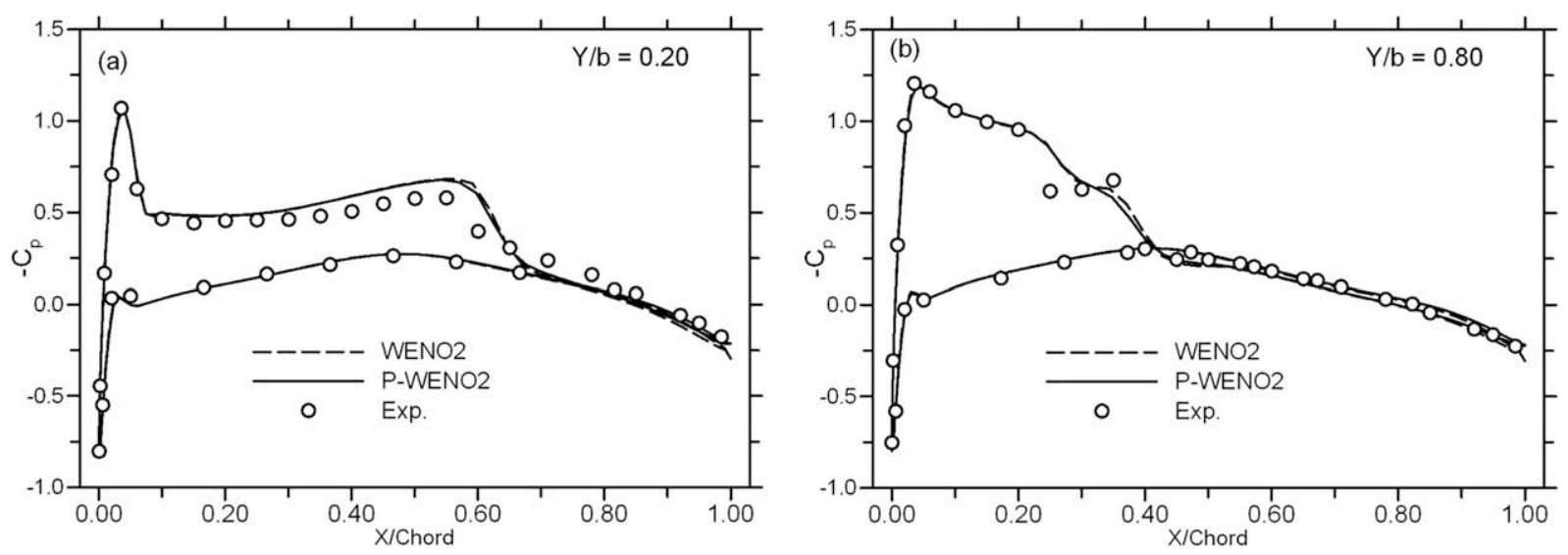

Fig. 17. Comparison of surface pressure distribution for ONERA M6 wing at $M_{\infty}=0.84, \alpha=3.06^{\circ}$ and $\operatorname{Re}_{\infty}=1.74 \times 10^{7}$; along spanwise chordline (a) $y / b=0.2$, (b) $y / b=0.8$, between P-WENO2 and WENO2 scheme.

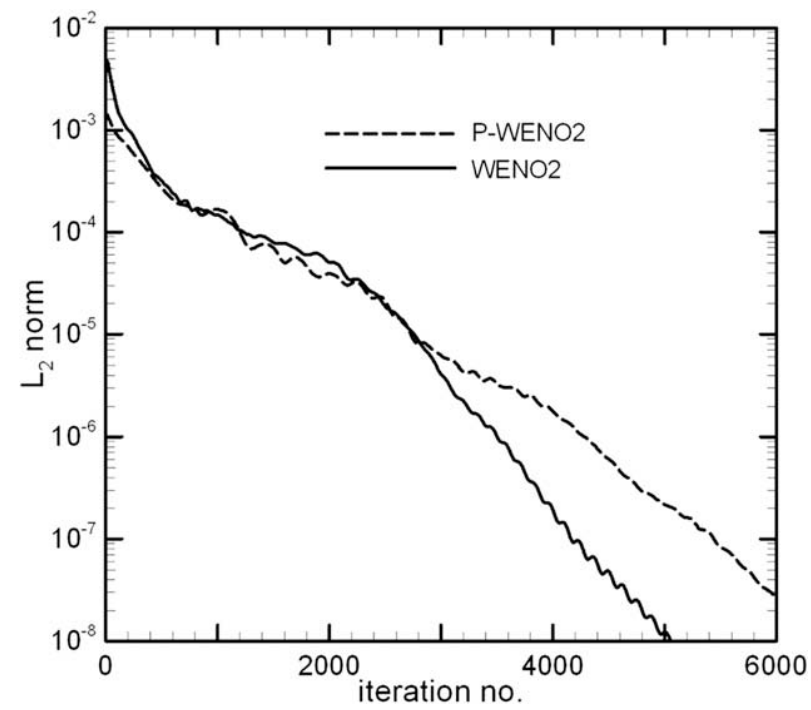

Fig. 18. Comparison of convergence history between P-WENO2 and WENO2 scheme for ONERA M6 wing at $M_{\infty}=0.84, \alpha=3.06^{\circ}$, and $R e_{\infty}=1.74 \times 10^{7}$.
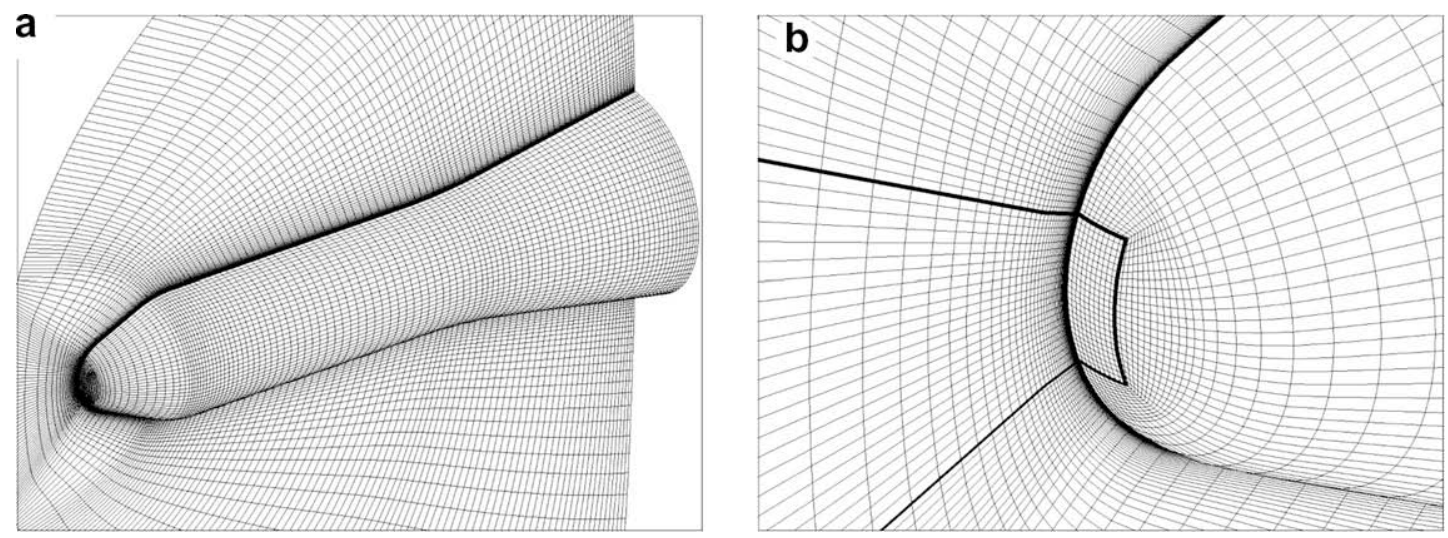

Fig. 19. A composite grid system of $\mathrm{C}-\mathrm{O}$ type and $\mathrm{H}-\mathrm{H}$ type grid topologies for $\mathrm{HB}-2$ model. 

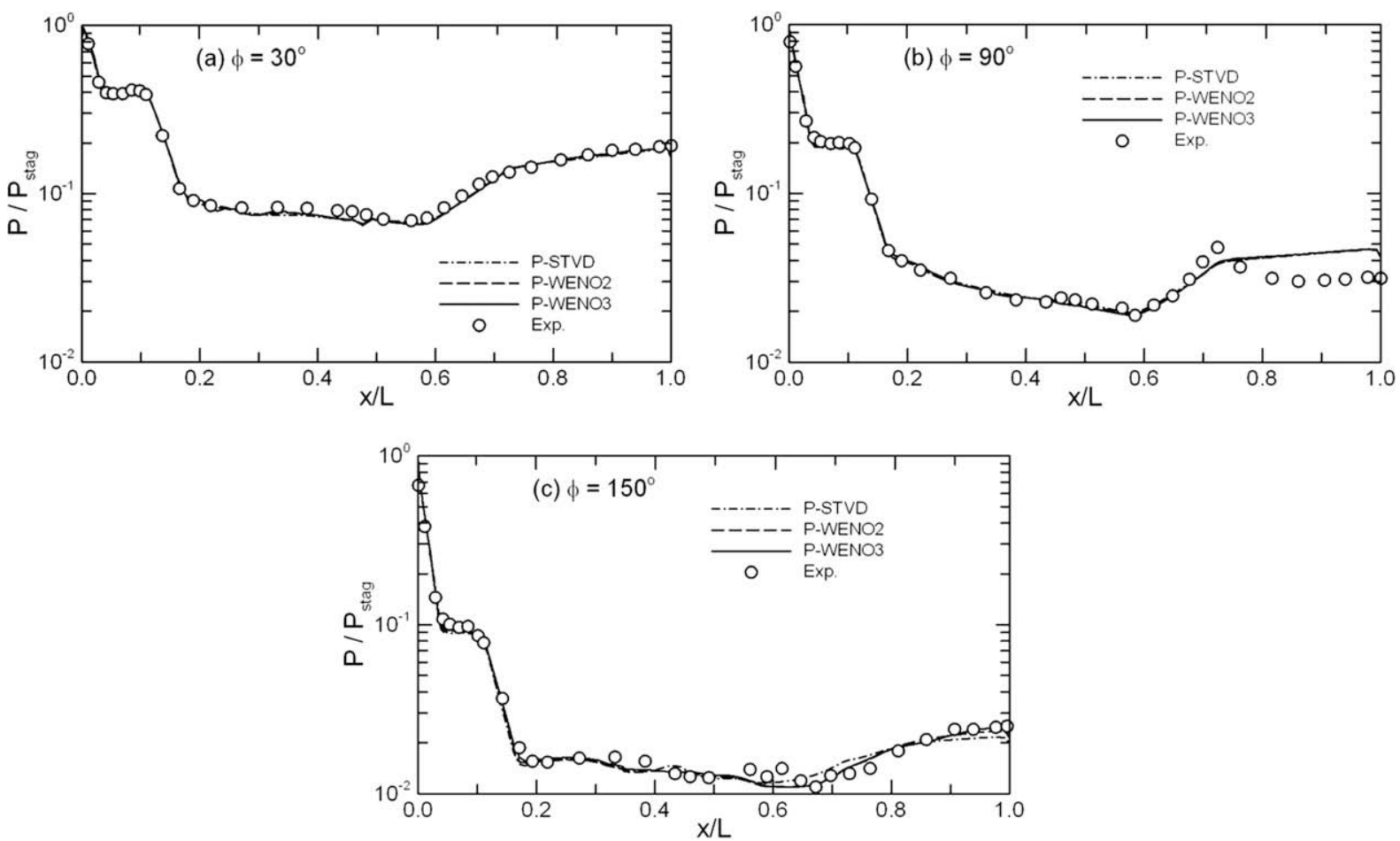

Fig. 20. Surface pressure distribution for HB-2 model at $M_{\infty}=5.1, \alpha=15^{\circ}$ and $R e_{\infty}=2.32 \times 10^{6}$; comparison of schemes at circumferential stations (a) $\phi=30^{\circ}$, (b) $\phi=90^{\circ}$ and (c) $\phi=150^{\circ}$.

\section{P-STVD}

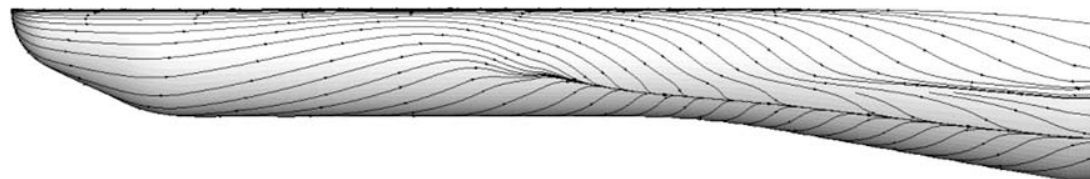

\section{P-WENO2}

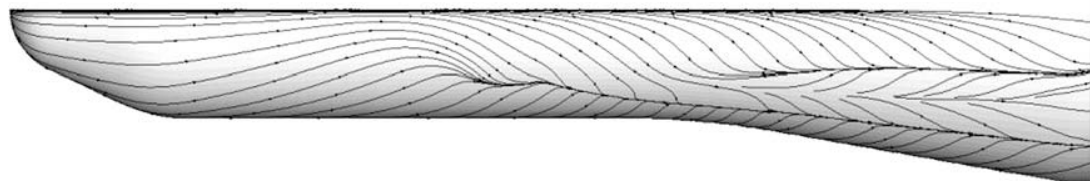

P-WENO3

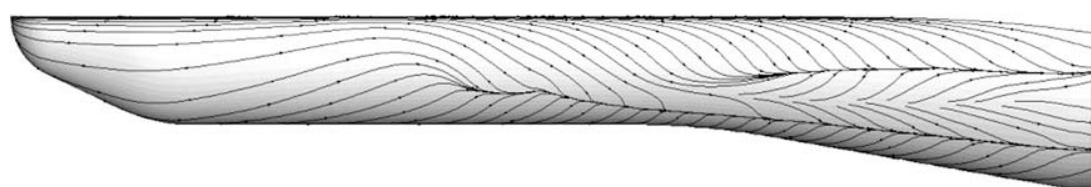

Fig. 21. Limiting streamline for HB-2 model by (a) P-STVD, (b) P-WENO2, and (c) P-WENO3 scheme, with the conditions $M_{\infty}=5.1, \alpha=15^{\circ}$, and $R e_{\infty}=2.32 \times 10^{6}$.

embedded oblique shock in front of flare, and shock boundary layer interactions. The solutions were calculated using PSTVD, P-WENO2 and P-WENO3 schemes. The surface pressure distributions in Fig. 20(a)-(c) show comparisons between experimental data by Gray and Lindsay [27] and computational results by all three schemes at various circumferential lines: 


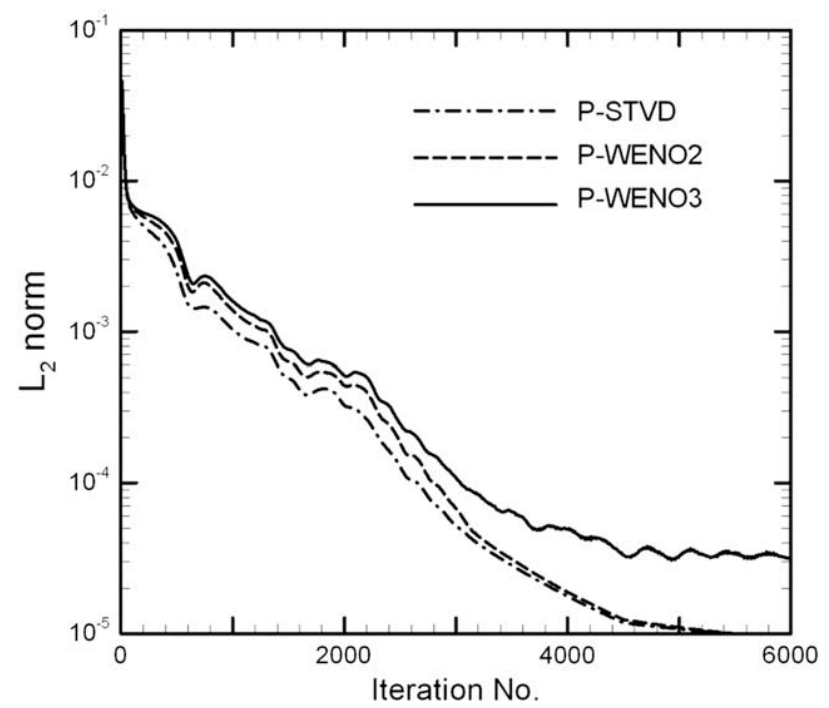

Fig. 22. Convergence history for HB-2 model at $M_{\infty}=5.1, \alpha=15^{\circ}$ and $R e_{\infty}=2.36 \times 10^{6}$.

$\phi=30^{\circ}, 90^{\circ}$ and $150^{\circ}$, respectively. For $\phi=30^{\circ}$, which is on the windward side, the computational results depict almost no differences between the three schemes and are in good agreement with experimental data. For $\phi=90^{\circ}$, which is the sideline of the body, the computational results also depict no differences between the three schemes, and are in a reasonable agreement with experimental data except for the rear part of the flare where the computational surface pressure is higher than the corresponding experimental data. It is suspected that the deviations might be attributed to the use of turbulence model which is less accurate in regards to separation flow. For $\phi=150^{\circ}$, which is on the leeward region of the body, the computational results by the three schemes also agree with each other except for the part near the front end of the flare where the shock boundary layer interaction occurs. The limiting streamlines in Fig. 21 show comparisons between the computational results by the three schemes. The topologies of limiting streamline, including the primary separation line, and re-attaching line and secondary separation line are shown. The positions of the primary separation line by P-WENO2 and P-WENO3 scheme agree well, but the limiting streamline by P-STVD scheme shows no secondary separation line and no clear reattaching line. It depicts the applicability of the high-order accurate schemes, P-WENO2 and P-WENO3, in solving the preconditioned system. The convergence histories are shown in Fig. 22. It shows that the P-STVD and P-WENO2 schemes have similar convergence history, they require 4500 iterations for three to four orders of magnitude. While for the P-WENO3 scheme, after three orders of magnitude converged, the convergence rate is slowing down.

\section{Conclusions}

Implicit high-resolution numerical codes for solving the preconditioned Navier-Stokes equations with Spalart-Allmaras one-equation turbulence model have been developed based on WENO methods and efficient lower-upper symmetric GaussSeidel implicit method. The resulting schemes have been successfully applied to the computations of various 2D/3D aerodynamic problems covering wide range of flow Mach numbers. The present scheme is a direct extension of the implicit WENO schemes for Navier-Stokes equation by Yang et al. [11] to the preconditioned Navier-Stokes system such that the stiff and slow convergence problem at low Mach number flows of the unconditioned system can be overcome. A discriminant for automatically adjusting both the preconditioning parameters at low Mach numbers and switching off the preconditioning at intermediate or high Mach numbers has also been introduced. Applications to lid driven cavity flow, low subsonic viscous flow over S809 airfoil, low speed viscous flow over a prolate spheroid, transonic flow over ONERA-M6 wing, and hypersonic flow over HB-2 model have been carried out to validate and illustrate the codes. It is found that, for all the cases computed, the solutions of the present algorithms are in very good agreement with the available experimental data. The combined use of preconditioned Navier-Stokes system and the WENO methods not only allows us to compute flows at all speeds but also enhances the accuracy of shock capturing for problems with shocks and improves the accuracy of low Mach number flows with complicated smooth flow structures.

\section{Acknowledgments}

This work was done under the auspices of National Science Council, Taiwan through Grant NSC 95-2212E002-055MY2. The authors wish to thank the reviewers for many constructive comments and suggestions. 


\section{References}

[1] J.S. Shuen, Y. Choi, K.H. Chen, A coupled implicit method for chemical non-equilibrium flows at all speeds, J. Comp. Phys. 106 (1994) $200-212$.

[2] E. Turkel, Robust Low Speed Preconditioning for Viscous High Lift Flows, AIAA Paper 2002-0962.

[3] L.D. Dailey, R.H. Pletcher, Evaluation of Multigrid Acceleration for Preconditioned Time-Accurate Navier-Stokes Algorithms, AIAA Paper 95-1668-CP.

[4] J.M. Weiss, W.A. Smith, Preconditioning applied to variable and constant density flows, AIAA J. 33 (11) (1995) $2050-2057$.

[5] D. Jespersen, T.H. Pulliam, P. Buning, Recent Enhancements to OVERFLOW, AIAA Paper 97-0644.

[6] S.A. Pandya, S. Venkateswaran, T.H. Pulliam, Implementation of Preconditioned Dual-time Procedures in OVERFLOW, AIAA Paper $2003-0072$.

[7] J.R. Edwards, C.J. Roy, Preconditioned multigrid methods for two-dimensional combustion calculation at all speeds, AIAA J. 35 (2) (1998) 185-192.

[8] X.-D. Liu, S. Osher, T. Chan, Weighted essentially nonoscillatory schemes, J. Comp. Phys. 115 (1994) $200-212$.

[9] G.-S. Jiang, C.-W. Shu, Efficient implementation of weighted ENO schemes, J. Comp. Phys. 126 (1996) $202-228$.

[10] J.-Y. Yang, S.-C. Yang, Y.-N. Chen, C.-A. Hsu, Weighted ENO schemes for the three-dimensional imcompressible Navier-Stokes equations, J. Comp. Phys. 146 (1998) 464-487.

[11] J.-Y. Yang, Y.-C. Perng, R.-H. Yen, Implicit weighted essentially nonoscillatory schemes for the compressible Navier-Stokes equations, AIAA J. 39 (11) (2001) 2082-2090.

[12] J.-Y. Yang, R.-H. Yen, Y.-C. Perng, Three-dimensional wing flow computations using implicit WENO Euler solver, J. of Aircraft 38 (6) (2001) 181-184.

[13] P.R. Spalart, S.R. Allmaras, A One-equation Turbulence Model for Aerodynamic Flows, AIAA Paper 92-257.

[14] J.E. Bardina, P.G. Huang, T.J. Coakley, Turbulence Modeling Validation, Testing and Development, NASA-TM-110446, April 1997.

[15] P.L. Roe, Approximate Riemann solvers, parameter vectors and difference, schemes, J. Comp. Phys. 43 (2) (1981) $357-372$.

[16] H.C. Yee, Construction of explicit and implicit symmetric TVD schemes and their applications, J. Comp. Phys. 68 (1987) 151-179.

[17] A.K. Henrick, T.D. Aslam, J.M. Powers, Mapped weighted essentially non-oscillatory schemes: achieving optimal order near critical points, J. Comp. Phys. 207 (2005) 542-567.

[18] C.M. Hung, W. Kordulla, A Time-split Finite-volume Algorithm for Three-dimensional Flow-field Simulation, AIAA Paper 83-1957.

[19] T.J. Coakley, Turbulence Modeling Methods for the Compressible Navier-Stokes Equations, AIAA Paper 83-1693.

[20] T. Siikonen, An application of Roe's flux-difference splitting for $k-\epsilon$ turbulence model, Int. J. for Numer. Meth. Fluids 21 (1995) $1017-1039$.

[21] S. Yoon, D. Kwak, Implicit Navier-Stokes solver for three-dimensional compressible flows, AIAA J. 30 (11) (1992) $2653-2659$.

[22] U. Ghia, K.N. Ghia, C.T. Shin, High-Re solutions for incompressible flow using the Navier-Stokes equations and a multigrid method, J. Comp. Phys. 48 (1982) 387-411.

[23] D.M. Somers, Design and Experimental Results for the S809 Airfoil, NREL/SR-440-6918, 1997.

[24] H.U. Meier, T. Cebeci, Flow characteristics of a body of revolution at incidence, in: Third Symposium on Numerical and Physical Aspects of Aerodynamic Flows, Long Beach, CA, January 21-24, 1985.

[25] V. Schmitt, F. Charpin, Pressure Distributions on the ONERA-M6-Wing at Transonic Mach Numbers AGARD-AR-138 B1-1, May 1979.

[26] J.D. Gray, Summary Report on Aerodynamic Characteristics of Standard Models HB-1 and HB-2, AEDC-TDR-64-137, July 1964.

[27] J.D. Gray, E.E. Lindsay, Force Tests of Standard Hypervelocity Ballistic Models HB-1 and HB-2 at Mach 1.5 to 10, AEDC-TDR-64-246, November 1964. 